\title{
An approximate solution to the fixed point problems for an infinite family of asymptotically strictly pseudocontractive mappings in the intermediate sense, cocoercive quasivariational inclusions problems and mixed equilibrium problems in Hilbert spaces
}

Pattanapong Tianchai*

"Correspondence: pattana@mju.ac.th

Faculty of Science, Maejo University, Chiangmai, 50290, Thailand

\section{空 Springer}

\begin{abstract}
We introduce a new iterative scheme by modifying Mann's iteration method to find a common element for the set of common fixed points of an infinite family of asymptotically strictly pseudocontractive mappings in the intermediate sense, the set of solutions of the cocoercive quasivariational inclusions problems, and the set of solutions of the mixed equilibrium problems in Hilbert spaces. The strong convergence theorem of the iterative scheme to a common element of the three aforementioned sets is obtained based on the shrinking projection method which extends and improves that of Ezeora and Shehu (Thai J. Math. 9(2):399-409, 2011) and many others.
\end{abstract}

MSC: $46 \mathrm{CO} 5 ; 47 \mathrm{HO9} ; 47 \mathrm{H} 10 ; 49 J 30 ; 49 J 40$

Keywords: fixed point; asymptotically strictly pseudocontractive in the intermediate sense; variational inequalities; mixed equilibrium; strong convergence; Hilbert space

\section{Introduction}

Throughout this paper, we always assume that $C$ is a nonempty closed convex subset of a real Hilbert space $H$ with inner product and norm denoted by $\langle\cdot, \cdot\rangle$ and $\|\cdot\|$, respectively. For a sequence $\left\{x_{n}\right\}$ in $H$, we denote the strong convergence and the weak convergence of $\left\{x_{n}\right\}$ to $x \in H$ by $x_{n} \rightarrow x$ and $x_{n} \rightarrow x$, respectively.

Recall that $P_{C}$ is the metric projection of $H$ onto $C$; that is, for each $x \in H$, there exists the unique point $P_{C} x \in C$ such that $\left\|x-P_{C} x\right\|=\min _{y \in C}\|x-y\|$. A mapping $T: C \rightarrow C$ is called nonexpansive if $\|T x-T y\| \leq\|x-y\|$ for all $x, y \in C$, and uniformly L-Lipschitzian if there exists a constant $L>0$ such that for each $n \in \mathbb{N},\left\|T^{n} x-T^{n} y\right\| \leq L\|x-y\|$ for all $x, y \in C$, and a mapping $f: C \rightarrow C$ is called a contraction if there exists a constant $\alpha \in(0,1)$ such that $\|f(x)-f(y)\| \leq \alpha\|x-y\|$ for all $x, y \in C$. A point $x \in C$ is a fixed point of $T$ provided that $T x=x$. We denote by $F(T)$ the set of fixed points of $T$; that is, $F(T)=\{x \in C: T x=x\}$. If $C$ 
is a nonempty bounded closed convex subset of $H$ and $T$ is a nonexpansive mapping of $C$ into itself, then $F(T)$ is nonempty (see [1]). Recall that a mapping $T: C \rightarrow C$ is said to be

(i) monotone if

$$
\langle T x-T y, x-y\rangle \geq 0, \quad \forall x, y \in C,
$$

(ii) $k$-Lipschitz continuous if there exists a constant $k>0$ such that

$$
\|T x-T y\| \leq k\|x-y\|, \quad \forall x, y \in C,
$$

if $k=1$, then $A$ is nonexpansive,

(iii) $\alpha$-strongly monotone if there exists a constant $\alpha>0$ such that

$$
\langle T x-T y, x-y\rangle \geq \alpha\|x-y\|^{2}, \quad \forall x, y \in C,
$$

(iv) $\alpha$-inverse-strongly monotone (or $\alpha$-cocoercive) if there exists a constant $\alpha>0$ such that

$$
\langle T x-T y, x-y\rangle \geq \alpha\|T x-T y\|^{2}, \quad \forall x, y \in C,
$$

if $\alpha=1$, then $T$ is called firmly nonexpansive; it is obvious that any $\alpha$-inverse-strongly monotone mapping $T$ is monotone and ( $1 / \alpha)$-Lipschitz continuous,

(v) $\kappa$-strictly pseudocontractive [2] if there exists a constant $\kappa \in[0,1)$ such that

$$
\|T x-T y\|^{2} \leq\|x-y\|^{2}+\kappa\|(I-T) x-(I-T) y\|^{2}, \quad \forall x, y \in C .
$$

In brief, we use $\kappa$-SPC to denote $\kappa$-strictly pseudocontractive. It is obvious that $T$ is nonexpansive if and only if $T$ is 0 -SPC,

(vi) asymptotically $\kappa-S P C[3]$ if there exists a constant $\kappa \in[0,1)$ and a sequence $\left\{\gamma_{n}\right\}$ of nonnegative real numbers with $\lim _{n \rightarrow \infty} \gamma_{n}=0$ such that

$$
\left\|T^{n} x-T^{n} y\right\|^{2} \leq\left(1+\gamma_{n}\right)\|x-y\|^{2}+\kappa\left\|\left(I-T^{n}\right) x-\left(I-T^{n}\right) y\right\|^{2}, \quad \forall x, y \in C,
$$

for all $n \in \mathbb{N}$. If $\kappa=0$, then $T$ is asymptotically nonexpansive with $k_{n}=\sqrt{1+\gamma_{n}}$ for all $n \in \mathbb{N}$; that is, $T$ is asymptotically nonexpansive [4] if there exists a sequence $\left\{k_{n}\right\} \subset[1, \infty)$ with $\lim _{n \rightarrow \infty} k_{n}=1$ such that

$$
\left\|T^{n} x-T^{n} y\right\| \leq k_{n}\|x-y\| \leq\left(1+\gamma_{n}\right)\|x-y\|, \quad \forall x, y \in C,
$$

for all $n \in \mathbb{N}$. It is known that the class of $\kappa$-SPC mappings and the class of asymptotically $\kappa$-SPC mappings are independent (see [5]). And the class of asymptotically nonexpansive mappings is reduced to the class of asymptotically nonexpansive mappings in the intermediate sense with $\epsilon_{n}=\gamma_{n} K$ for all $n \in \mathbb{N}$ and for some $K>0$; that is, $T$ is an asymptotically nonexpansive mapping in the intermediate sense if there exists a sequence $\left\{\epsilon_{n}\right\}$ of nonnegative real numbers with $\lim _{n \rightarrow \infty} \epsilon_{n}=0$ such that

$$
\left\|T^{n} x-T^{n} y\right\| \leq\|x-y\|+\epsilon_{n}, \quad \forall x, y \in C,
$$

for all $n \in \mathbb{N}$, 
(vii) asymptotically $\kappa-S P C$ mapping in the intermediate sense [6] if there exists a constant $\kappa \in[0,1)$ and a sequence $\left\{\gamma_{n}\right\}$ of nonnegative real numbers with $\lim _{n \rightarrow \infty} \gamma_{n}=0$ such that

$$
\limsup _{n \rightarrow \infty} \sup _{x, y \in C}\left(\left\|T^{n} x-T^{n} y\right\|^{2}-\left(1+\gamma_{n}\right)\|x-y\|^{2}-\kappa\left\|\left(I-T^{n}\right) x-\left(I-T^{n}\right) y\right\|^{2}\right) \leq 0 .
$$

If we define

$$
\tau_{n}=\max \left\{0, \sup _{x, y \in C}\left(\left\|T^{n} x-T^{n} y\right\|^{2}-\left(1+\gamma_{n}\right)\|x-y\|^{2}-\kappa\left\|\left(I-T^{n}\right) x-\left(I-T^{n}\right) y\right\|^{2}\right)\right\},
$$

then $\lim _{n \rightarrow \infty} \tau_{n}=0$, and the last inequality is reduced to

$$
\left\|T^{n} x-T^{n} y\right\|^{2} \leq\left(1+\gamma_{n}\right)\|x-y\|^{2}+\kappa\left\|\left(I-T^{n}\right) x-\left(I-T^{n}\right) y\right\|^{2}+\tau_{n}, \quad \forall x, y \in C,
$$

for all $n \in \mathbb{N}$. It is obvious that if $\tau_{n}=0$ for all $n \in \mathbb{N}$, then the class of asymptotically $\kappa$-SPC mappings in the intermediate sense is reduced to the class of asymptotically $\kappa$-SPC mappings; and if $\tau_{n}=\kappa=0$ for all $n \in \mathbb{N}$, then the class of asymptotically $\kappa$-SPC mappings in the intermediate sense is reduced to the class of asymptotically nonexpansive mappings; and if $\gamma_{n}=\tau_{n}=\kappa=0$ for all $n \in \mathbb{N}$, then the class of asymptotically $\kappa$-SPC mappings in the intermediate sense is reduced to the class of nonexpansive mappings; and the class of asymptotically nonexpansive mappings in the intermediate sense with $\left\{\epsilon_{n}\right\}$ of nonnegative real numbers such that $\lim _{n \rightarrow \infty} \epsilon_{n}=0$ is reduced to the class of asymptotically $\kappa$-SPC mappings in the intermediate sense with $\tau_{n}=\epsilon_{n} K$ for all $n \in \mathbb{N}$ and for some $K>0$. Some methods have been proposed to solve the fixed point problem of an asymptotically $\kappa$-SPC mapping in the intermediate sense (1.9); related work can also be found in [6-13] and the references therein.

Example 1.1 (Sahu et al. [6]) Let $X=\mathbb{R}$ and $C=[0,1]$. For each $x \in C$, we define $T: C \rightarrow C$ by

$$
T x= \begin{cases}\kappa x, & \text { if } x \in\left[0, \frac{1}{2}\right], \\ 0, & \text { if } x \in\left(\frac{1}{2}, 1\right]\end{cases}
$$

where $\kappa \in(0,1)$. Then

(1) $T$ is an asymptotically nonexpansive mapping in the intermediate sense. Therefore, $T$ is an asymptotically $\kappa$-SPC mapping in the intermediate sense.

(2) $T$ is not continuous. Therefore, $T$ is not an asymptotically $\kappa-\mathrm{SPC}$ and asymptotically nonexpansive mapping.

Example 1.2 (Hu and Cai [7]) Let $X=\mathbb{R}, C=[0,1]$, and $\kappa \in[0,1)$. For each $x \in C$, we define $T: C \rightarrow C$ by

$$
T x= \begin{cases}\frac{1}{4} \sqrt{\frac{1}{2}-x}+\frac{\sqrt{2}}{2}, & \text { if } x \in\left[0, \frac{1}{2}\right], \\ \sqrt{x}, & \text { if } x \in\left(\frac{1}{2}, 1\right] .\end{cases}
$$

Then 
(1) $T$ is an asymptotically nonexpansive mapping in the intermediate sense. Therefore, $T$ is an asymptotically $\kappa$-SPC mapping in the intermediate sense.

(2) $T$ is continuous but not uniformly $L$-Lipschitzian. Therefore, $T$ is not an asymptotically $\kappa$-SPC mapping.

Example 1.3 Let $X=\mathbb{R}$ and $C=[0,1]$. For each $x \in C$, we define $T: C \rightarrow C$ by

$$
T x= \begin{cases}\sqrt{\frac{2 x+1}{4}}-\frac{1}{2}, & \text { if } x \in\left[0, \frac{1}{2}\right], \\ \frac{2 \kappa x}{5}, & \text { if } x \in\left(\frac{1}{2}, 1\right],\end{cases}
$$

where $\kappa \in[0,1)$. Then

(1) $T$ is an asymptotically nonexpansive mapping in the intermediate sense. Therefore, $T$ is an asymptotically $\kappa$-SPC mapping in the intermediate sense.

(2) $T$ is not continuous. Therefore, $T$ is not an asymptotically $\kappa-\mathrm{SPC}$ and asymptotically nonexpansive mapping.

Iterative methods are often used to solve the fixed point equation $T x=x$. The most wellknown method is perhaps the Picard successive iteration method when $T$ is a contraction. Picard's method generates a sequence $\left\{x_{n}\right\}$ successively as $x_{n+1}=T x_{n}$ for all $n \in \mathbb{N}$ with $x_{1}=x$ chosen arbitrarily, and this sequence converges in norm to the unique fixed point of $T$. However, if $T$ is not a contraction (for instance, if $T$ is nonexpansive), then Picard's successive iteration fails, in general, to converge. Instead, Mann's iteration method for a nonexpansive mapping $T$ (see [14]) prevails, generates a sequence $\left\{x_{n}\right\}$ recursively by

$$
x_{n+1}=\alpha_{n} x_{n}+\left(1-\alpha_{n}\right) T x_{n}, \quad \forall n \in \mathbb{N},
$$

where $x_{1}=x \in C$ chosen arbitrarily and the sequence $\left\{\alpha_{n}\right\}$ lies in the interval $[0,1]$.

Mann's algorithm for nonexpansive mappings has been extensively investigated (see $[2,15,16]$ and the references therein). One of the well-known results is proven by $\operatorname{Re}-$ ich [16] for a nonexpansive mapping $T$ on $C$, which asserts the weak convergence of the sequence $\left\{x_{n}\right\}$ generated by (1.10) in a uniformly convex Banach space with a Frechet differentiable norm under the control condition $\sum_{n=1}^{\infty} \alpha_{n}\left(1-\alpha_{n}\right)=\infty$. Recently, Marino and $\mathrm{Xu}$ [17] developed and extended Reich's result to a SPC mapping in a Hilbert space setting. More precisely, they proved the weak convergence of Mann's iteration process (1.10) for a $\kappa$-SPC mapping $T$ on $C$, and subsequently, this result was improved and carried over the class of asymptotically $\kappa$-SPC mappings by Kim and Xu [18].

It is known that Mann's iteration (1.10) is in general not strongly convergent (see [19]). The way to guarantee strong convergence has been proposed by Nakajo and Takahashi [20]. They modified Mann's iteration method (1.10), which is to find a fixed point of a nonexpansive mapping by the hybrid method, called the shrinking projection method (or the CQ method), as the following theorem.

Theorem NT Let $C$ be a nonempty closed convex subset of a real Hilbert space H. Let $T$ be a nonexpansive mapping of $C$ into itself such that $F(T) \neq \emptyset$. Suppose that $x_{1}=x \in C$ chosen 
arbitrarily and $\left\{x_{n}\right\}$ is the sequence defined by

$$
\left\{\begin{array}{l}
y_{n}=\alpha_{n} x_{n}+\left(1-\alpha_{n}\right) T x_{n}, \\
C_{n}=\left\{z \in C:\left\|y_{n}-z\right\| \leq\left\|x_{n}-z\right\|\right\}, \\
Q_{n}=\left\{z \in C:\left\langle x_{n}-z, x_{1}-x_{n}\right\rangle \geq 0\right\} \\
x_{n+1}=P_{C_{n} \cap Q_{n}}\left(x_{1}\right), \quad \forall n \in \mathbb{N},
\end{array}\right.
$$

where $0 \leq \alpha_{n} \leq \alpha<1$. Then $\left\{x_{n}\right\}$ converges strongly to $P_{F(T)}\left(x_{1}\right)$.

Subsequently, Marino and $\mathrm{Xu}$ [21] introduced an iterative scheme for finding a fixed point of a $\kappa$-SPC mapping as the following theorem.

Theorem MX Let $C$ be a nonempty closed convex subset of a real Hilbert space $H$ and let $T: C \rightarrow C$ be a $\kappa$-SPC mapping for some $0 \leq \kappa<1$. Assume that $F(T) \neq \emptyset$. Suppose that $x_{1}=x \in C$ chosen arbitrarily and $\left\{x_{n}\right\}$ is the sequence defined by

$$
\left\{\begin{array}{l}
y_{n}=\alpha_{n} x_{n}+\left(1-\alpha_{n}\right) T x_{n}, \\
C_{n}=\left\{z \in C:\left\|y_{n}-z\right\|^{2} \leq\left\|x_{n}-z\right\|^{2}+\left(1-\alpha_{n}\right)\left(\kappa-\alpha_{n}\right)\left\|x_{n}-T x_{n}\right\|^{2}\right\}, \\
Q_{n}=\left\{z \in C:\left\langle x_{n}-z, x_{1}-x_{n}\right\rangle \geq 0\right\}, \\
x_{n+1}=P_{C_{n} \cap Q_{n}}\left(x_{1}\right), \quad \forall n \in \mathbb{N},
\end{array}\right.
$$

where $0 \leq \alpha_{n}<1$. Then the sequence $\left\{x_{n}\right\}$ converges strongly to $P_{F(T)}\left(x_{1}\right)$.

Quite recently, Kim and Xu [18] have improved and carried Theorem MX over a wider class of asymptotically $\kappa$-SPC mappings as the following theorem.

Theorem KX Let $C$ be a nonempty closed convex subset of a real Hilbert space $H$ and let $T: C \rightarrow C$ be an asymptotically $\kappa$-SPC mapping for some $0 \leq \kappa<1$ with a bounded sequence $\left\{\gamma_{n}\right\} \subset[0, \infty)$ such that $\lim _{n \rightarrow \infty} \gamma_{n}=0$. Assume that $F(T)$ is a nonempty bounded subset of $C$. Suppose that $x_{1}=x \in C$ chosen arbitrarily and $\left\{x_{n}\right\}$ is the sequence defined by

$$
\left\{\begin{array}{l}
y_{n}=\alpha_{n} x_{n}+\left(1-\alpha_{n}\right) T^{n} x_{n}, \\
C_{n}=\left\{z \in C:\left\|y_{n}-z\right\|^{2} \leq\left\|x_{n}-z\right\|^{2}+\left(\kappa-\alpha_{n}\left(1-\alpha_{n}\right)\right)\left\|x_{n}-T^{n} x_{n}\right\|^{2}+\theta_{n}\right\}, \\
Q_{n}=\left\{z \in C:\left\langle x_{n}-z, x_{1}-x_{n}\right\rangle \geq 0\right\}, \\
x_{n+1}=P_{C_{n} \cap Q_{n}}\left(x_{1}\right), \quad \forall n \in \mathbb{N},
\end{array}\right.
$$

where $\theta_{n}=\Delta_{n}^{2}\left(1-\alpha_{n}\right) \gamma_{n} \rightarrow 0$ as $n \rightarrow \infty, \Delta_{n}=\sup \left\{\left\|x_{n}-z\right\|: z \in F(T)\right\}<\infty$ and $0 \leq \alpha_{n}<1$ such that $\lim _{\sup _{n \rightarrow \infty}} \alpha_{n}<1-\kappa$. Then the sequence $\left\{x_{n}\right\}$ converges strongly to $P_{F(T)}\left(x_{1}\right)$.

The domain of the function $\varphi: C \rightarrow \mathbb{R} \cup\{+\infty\}$ is the set

$$
\operatorname{dom} \varphi=\{x \in C: \varphi(x)<+\infty\}
$$

Let $\varphi: C \rightarrow \mathbb{R} \cup\{+\infty\}$ be a proper extended real-valued function and let $\Phi$ be a bifunction from $C \times C$ into $\mathbb{R}$, where $\mathbb{R}$ is the set of real numbers. The so-called mixed equilibrium 
problem is to find $x \in C$ such that

$$
\Phi(x, y)+\varphi(y)-\varphi(x) \geq 0, \quad \forall y \in C
$$

The set of solutions of problem (1.11) is denoted by $\operatorname{MEP}(\Phi, \varphi)$, that is,

$$
\operatorname{MEP}(\Phi, \varphi)=\{x \in C: \Phi(x, y)+\varphi(y)-\varphi(x) \geq 0, \forall y \in C\}
$$

It is obvious that if $x$ is a solution of problem (1.11), then $x \in \operatorname{dom} \varphi$. If $\varphi=0$, then problem (1.11) is reduced to finding $x \in C$ such that

$$
\Phi(x, y) \geq 0, \quad \forall y \in C
$$

We denote by $\operatorname{EP}(\Phi)$ the set of solutions of the equilibrium problem. The theory of equilibrium problems has played an important role in the study of a wide class of problems arising in economics, finance, transportation, network and structural analysis, elasticity, and optimization and has numerous applications, including but not limited to problems in economics, game theory, finance, traffic analysis, circuit network analysis, and mechanics. The ideas and techniques of this theory are being used in a variety of diverse areas and have proved to be productive and innovative. Problem (1.12) is very general in the sense that it includes, as special cases, optimization problems, variational inequalities, minimax problems, the Nash equilibrium problem in noncooperative games, and others; see, for instance, $[22,23]$ and the references therein. Some methods have been proposed to solve equilibrium problem (1.12); related work can also be found in [7, 12, 24-34].

For solving the mixed equilibrium problem, let us assume that the bifunction $\Phi: C \times$ $C \rightarrow \mathbb{R}$, the function $\varphi: C \rightarrow \mathbb{R} \cup\{+\infty\}$, and the set $C$ satisfy the following conditions:

(A1) $\Phi(x, x)=0$ for all $x \in C$;

(A2) $\Phi$ is monotone; that is, $\Phi(x, y)+\Phi(y, x) \leq 0$ for all $x, y \in C$;

(A3) for each $x, y, z \in C$,

$$
\lim _{t \downarrow 0} \Phi(t z+(1-t) x, y) \leq \Phi(x, y)
$$

(A4) for each $x \in C, y \mapsto \Phi(x, y)$ is convex and lower semicontinuous;

(A5) for each $y \in C, x \mapsto \Phi(x, y)$ is weakly upper semicontinuous;

(B1) for each $x \in C$ and $r>0$, there exists a bounded subset $D_{x} \subset C$ and $y_{x} \in C$ such that for any $z \in C \backslash D_{x}$,

$$
\Phi\left(z, y_{x}\right)+\varphi\left(y_{x}\right)-\varphi(z)+\frac{1}{r}\left\langle y_{x}-z, z-x\right\rangle<0
$$

(B2) $C$ is a bounded set.

Variational inequality theory provides us with a simple, natural, general, and unified framework for studying a wide class of unrelated problems arising in elasticity, structural analysis, economics, optimization, oceanography, and regional, physical, and engineering sciences, etc. (see [35-41] and the references therein). In recent years, variational inequalities have been extended and generalized in different directions, using novel and innovative techniques, both for their own sake and for their applications. A useful and important generalization of variational inequalities is a variational inclusion. 
Let $B: H \rightarrow H$ be a single-valued nonlinear mapping and let $M: H \rightarrow 2^{H}$ be a set-valued mapping. We consider the following quasivariational inclusion problem, which is to find a point $x \in H$ such that

$$
\theta \in B x+M x
$$

where $\theta$ is the zero vector in $H$. The set of solutions of problem (1.13) is denoted by $V I(H, B, M)$.

A set-valued mapping $T: H \rightarrow 2^{H}$ is called monotone if for all $x, y \in H, f \in T x$ and $g \in T y$ imply $\langle x-y, f-g\rangle \geq 0$. A monotone mapping $T: H \rightarrow 2^{H}$ is maximal if the graph of $G(T)$ of $T$ is not properly contained in the graph of any other monotone mappings. It is known that a monotone mapping $T$ is maximal if and only if for $(x, f) \in H \times H,\langle x-y, f-g\rangle \geq 0$ for all $(y, g) \in G(T)$ implies $f \in T x$.

Definition 1.4 (see [42]) Let $M: H \rightarrow 2^{H}$ be a multi-valued maximal monotone mapping. Then the single-valued mapping $J_{M, \lambda}: H \rightarrow H$ defined by $J_{M, \lambda}(u)=(I+\lambda M)^{-1}(u)$, for all $u \in H$, is called the resolvent operator associated with $M$, where $\lambda$ is any positive number and $I$ is the identity mapping.

Recently, Qin et al. [43] introduced the following algorithm for a finite family of asymptotically $\kappa_{i}$-SPC mappings $T_{i}$ on $C$. Let $x_{0} \in C$ and $\left\{\alpha_{n}\right\}_{n=0}^{\infty}$ be a sequence in $[0,1]$. Let $N \geq 1$ be an integer. The sequence $\left\{x_{n}\right\}$ generated in the following way:

$$
\begin{aligned}
& x_{1}=\alpha_{0} x_{0}+\left(1-\alpha_{0}\right) T_{1} x_{0}, \\
& x_{2}=\alpha_{1} x_{1}+\left(1-\alpha_{1}\right) T_{2} x_{1}, \\
& \vdots \\
& x_{N}=\alpha_{N-1} x_{N-1}+\left(1-\alpha_{N-1}\right) T_{N} x_{N-1}, \\
& x_{N+1}=\alpha_{N} x_{N}+\left(1-\alpha_{N}\right) T_{1}^{2} x_{N}, \\
& \vdots \\
& x_{2 N}=\alpha_{2 N-1} x_{2 N-1}+\left(1-\alpha_{2 N-1}\right) T_{N}^{2} x_{2 N-1}, \\
& x_{2 N+1}=\alpha_{2 N} x_{2 N}+\left(1-\alpha_{2 N}\right) T_{N}^{3} x_{2 N},
\end{aligned}
$$

is called the explicit iterative scheme of a finite family of asymptotically $\kappa_{i}$-SPC mappings $T_{i}$ on $C$, where $i=1,2, \ldots, N$. Since, for each $n \in \mathbb{N}$, it can be written as $n=(h-1) N+i$, where $i=i(n) \in\{1,2, \ldots, N\}, h=h(n) \geq 1$ is a positive integer and $h(n) \rightarrow \infty$ as $n \rightarrow \infty$.

For each $i=1,2, \ldots, N$, let $\left\{T_{i}: C \rightarrow C\right\}$ be a finite family of asymptotically $\kappa_{i}$-SPC mappings with the sequence $\left\{\gamma_{n, i}\right\}_{n=1}^{\infty} \subset[0, \infty)$ such that $\lim _{n \rightarrow \infty} \gamma_{n, i}=0$. One has

$$
\begin{aligned}
\left\|T_{i(n)}^{h(n)} x-T_{i(n)}^{h(n)} y\right\|^{2} \leq & \left(1+\gamma_{h(n), i(n)}\right)\|x-y\|^{2} \\
& +\kappa_{i(n)}\left\|\left(I-T_{i(n)}^{h(n)}\right) x-\left(I-T_{i(n)}^{h(n)}\right) y\right\|^{2}, \quad \forall x, y \in C,
\end{aligned}
$$


for all $n \in \mathbb{N}$, and we can rewrite (1.14) in the following compact form:

$$
x_{n}=\alpha_{n-1} x_{n-1}+\left(1-\alpha_{n-1}\right) T_{i(n)}^{h(n)} x_{n-1}, \quad \forall n \in \mathbb{N} .
$$

To be more precise, they introduced an iterative scheme for finding a common fixed point of a finite family of asymptotically $\kappa_{i}$-SPC mappings as the following theorem.

Theorem QCKS Let C be a nonempty closed convex subset of a real Hilbert space H. Let $N \geq 1$ be an integer. For each $i=1,2, \ldots, N$, let $\left\{T_{i}: C \rightarrow C\right\}$ be a finite family of asymptotically $\kappa_{i}$-SPC mappings defined as in (1.15), when $\kappa_{i} \in[0,1)$ with the sequence $\left\{\gamma_{n, i}\right\} \subset[0, \infty)$ such that $\lim _{n \rightarrow \infty} \gamma_{n, i}=0$. Let $\kappa=\max \left\{\kappa_{i}: 1 \leq i \leq N\right\}$ and $\gamma_{n}=\max \left\{\sqrt{1+\gamma_{n, i}}: 1 \leq i \leq N\right\}$. Assume that $\Omega:=\bigcap_{i=1}^{N} F\left(T_{i}\right)$ is a nonempty bounded subset of $C$. For $x_{0}=x \in C$ chosen arbitrarily, suppose that $\left\{x_{n}\right\}$ is generated iteratively by

$$
\left\{\begin{aligned}
& y_{n-1}= \alpha_{n-1} x_{n-1}+\left(1-\alpha_{n-1}\right) T_{i(n)}^{h(n)} x_{n-1}, \\
& C_{n-1}=\left\{z \in C:\left\|y_{n-1}-z\right\|^{2} \leq\left\|x_{n-1}-z\right\|^{2}+\theta_{n-1}\right. \\
&\left.\quad-\left(1-\alpha_{n-1}\right)\left(\alpha_{n-1}-\kappa\right)\left\|T_{i(n)}^{h(n)} x_{n-1}-x_{n-1}\right\|^{2}\right\}, \\
& Q_{n-1}=\left\{z \in C:\left\langle x_{n-1}-z, x_{0}-x_{n-1}\right\rangle \geq 0\right\}, \\
& x_{n}=P_{C_{n-1} \cap Q_{n-1}}\left(x_{0}\right), \quad \forall n \in \mathbb{N},
\end{aligned}\right.
$$

where $\theta_{n-1}=\left(\gamma_{h(n)}^{2}-1\right)\left(1-\alpha_{n-1}\right) \cdot \Delta_{n-1}^{2} \rightarrow 0$ as $n \rightarrow \infty$ such that $\Delta_{n-1}=\sup \left\{\left\|x_{n-1}-z\right\|:\right.$ $z \in \Omega\}<\infty$. If the control sequence $\left\{\alpha_{n}\right\}_{n=0}^{\infty}$ is chosen such that $0 \leq \alpha_{n} \leq \alpha<1$. Then the sequence $\left\{x_{n}\right\}$ converges strongly to $P_{\Omega}\left(x_{0}\right)$.

Recall that a discrete family $\mathcal{S}=\left\{T_{n}: n \geq 0\right\}$ of self-mappings of $C$ is said to be an asymptotically $\kappa$-SPC semigroup [44] if the following conditions are satisfied:

(1) $T_{0}=I$, where $I$ denotes the identity operator on $C$;

(2) $T_{n+m} x=T_{n} T_{m} x, \forall n, m \geq 0, \forall x \in C$;

(3) there exists a constant $\kappa \in[0,1)$ and a sequence $\left\{\gamma_{n}\right\}$ of nonnegative real numbers with $\lim _{n \rightarrow \infty} \gamma_{n}=0$ such that

$$
\begin{aligned}
\left\|T_{n} x-T_{n} y\right\|^{2} \leq & \left(1+\gamma_{n}\right)\|x-y\|^{2} \\
& +\kappa\left\|\left(I-T_{n}\right) x-\left(I-T_{n}\right) y\right\|^{2}, \quad \forall x, y \in C,
\end{aligned}
$$

for all $n \geq 0$. Note that, for a single asymptotically $\kappa$-SPC mapping $T: C \rightarrow C$, (1.16) immediately reduces to (1.6) by taking $T_{n}=T^{n}$ for all $n \geq 0$ such that $T^{0}=I$.

On the other hand, Tianchai [24] introduced an iterative scheme for finding a common element of the set of solutions of the mixed equilibrium problems and the set of common fixed points for a discrete asymptotically $\kappa$-SPC semigroup which is a subclass of the class of infinite families for the asymptotically $\kappa$-SPC mapping as the following theorem.

Theorem $\mathbf{T}$ Let $C$ be a nonempty closed convex subset of a real Hilbert space $H$, $\Phi$ be a bifunction from $C \times C$ into $\mathbb{R}$ satisfying the conditions (A1)-(A5), and let $\varphi: C \rightarrow \mathbb{R} \cup\{+\infty\}$ be a proper lower semicontinuous and convex function with the assumption that either (B1) or (B2) holds. Let $\mathcal{S}=\left\{T_{n}: n \geq 0\right\}$ be an asymptotically $\kappa$-SPC semigroup on $C$ for some 
$\kappa \in[0,1)$ with the sequence $\left\{\gamma_{n}\right\} \subset[0, \infty)$ such that $\lim _{n \rightarrow \infty} \gamma_{n}=0$. Assume that $\Omega:=F(\mathcal{S}) \cap$ $\operatorname{MEP}(\Phi, \varphi)=\bigcap_{n=0}^{\infty} F\left(T_{n}\right) \cap \operatorname{MEP}(\Phi, \varphi)$ is a nonempty bounded subset of $C$. For $x_{0}=x \in C$ chosen arbitrarily, suppose that $\left\{x_{n}\right\},\left\{y_{n}\right\}$ and $\left\{u_{n}\right\}$ are generated iteratively by

$$
\left\{\begin{aligned}
u_{n} \in & C \text { such that } \\
& \Phi\left(u_{n}, y\right)+\varphi(y)-\varphi\left(u_{n}\right)+\frac{1}{r_{n}}\left\langle y-u_{n}, u_{n}-x_{n}\right\rangle \geq 0, \quad \forall y \in C, \\
y_{n}=\alpha_{n} u_{n}+\left(1-\alpha_{n}\right) T_{n} u_{n}, & \\
C_{n+1}= & \left\{z \in C_{n} \cap Q_{n}:\left\|y_{n}-z\right\|^{2} \leq\left\|x_{n}-z\right\|^{2}\right. \\
& \left.\quad\left(1-\alpha_{n}\right)\left(\theta_{n}+\left(\kappa-\alpha_{n}\right)\left\|u_{n}-T_{n} u_{n}\right\|^{2}\right)\right\}, \\
Q_{n+1}= & \left\{z \in C_{n} \cap Q_{n}:\left\langle x_{n}-z, x_{0}-x_{n}\right\rangle \geq 0\right\}, \\
C_{0}= & Q_{0}=C, \\
x_{n+1}= & P_{C_{n+1} \cap Q_{n+1}}\left(x_{0}\right), \quad \forall n \in \mathbb{N} \cup\{0\},
\end{aligned}\right.
$$

where $\theta_{n}=\gamma_{n} \cdot \sup \left\{\left\|x_{n}-z\right\|^{2}: z \in \Omega\right\}<\infty,\left\{\alpha_{n}\right\} \subset[a, b]$ such that $\kappa<a<b<1,\left\{r_{n}\right\} \subset[r, \infty)$ for some $r>0$ and $\sum_{n=0}^{\infty}\left|r_{n+1}-r_{n}\right|<\infty$. Then the sequences $\left\{x_{n}\right\},\left\{y_{n}\right\}$, and $\left\{u_{n}\right\}$ converge strongly to $w=P_{\Omega}\left(x_{0}\right)$

Recently, Saha et al. [6] modified Mann's iteration method (1.10) for finding a fixed point of the asymptotically $\kappa$-SPC mapping in the intermediate sense which is not necessarily uniformly Lipschitzian (see, e.g., $[6,7]$ ) as the following theorem.

Theorem SXY Let C be a nonempty closed convex subset of a real Hilbert space $H$ and let $T: C \rightarrow C$ be a uniformly continuous and asymptotically $\kappa$-SPC mapping in the intermediate sense defined as in (1.9), when $\kappa \in[0,1)$, with the sequences $\left\{\gamma_{n}\right\},\left\{\tau_{n}\right\} \subset[0, \infty)$ such that $\lim _{n \rightarrow \infty} \gamma_{n}=\lim _{n \rightarrow \infty} \tau_{n}=0$. Assume that $F(T)$ is a nonempty bounded subset of $C$. Suppose that $x_{1}=x \in C$ chosen arbitrarily and $\left\{x_{n}\right\}$ is the sequence defined by

$$
\left\{\begin{array}{l}
y_{n}=\left(1-\alpha_{n}\right) x_{n}+\alpha_{n} T^{n} x_{n}, \\
C_{n}=\left\{z \in C:\left\|y_{n}-z\right\|^{2} \leq\left\|x_{n}-z\right\|^{2}+\theta_{n}\right\}, \\
Q_{n}=\left\{z \in C:\left\langle x_{n}-z, x_{1}-x_{n}\right\rangle \geq 0\right\}, \\
x_{n+1}=P_{C_{n} \cap Q_{n}}\left(x_{1}\right), \quad \forall n \in \mathbb{N},
\end{array}\right.
$$

where $\theta_{n}=\gamma_{n} \cdot \Delta_{n}+\tau_{n} \rightarrow 0$ as $n \rightarrow \infty, \Delta_{n}=\sup \left\{\left\|x_{n}-z\right\|: z \in F(T)\right\}<\infty$ and $\left\{\alpha_{n}\right\} \subset(0,1]$ such that $0<\alpha \leq \alpha_{n} \leq 1-\kappa$. Then the sequence $\left\{x_{n}\right\}$ converges strongly to $P_{F(T)}\left(x_{1}\right)$.

Let $N \geq 1$ be an integer. For each $i=0,1, \ldots, N-1$, let $\left\{T_{i}: C \rightarrow C\right\}$ be a finite family of asymptotically $\kappa_{i}$-SPC mappings in the intermediate sense with the sequences $\left\{\gamma_{n, i}\right\}_{n=1}^{\infty},\left\{\tau_{n, i}\right\}_{n=1}^{\infty} \subset[0, \infty)$ such that $\lim _{n \rightarrow \infty} \gamma_{n, i}=\lim _{n \rightarrow \infty} \tau_{n, i}=0$. One has

$$
\begin{aligned}
\left\|T_{i(n)}^{h(n)} x-T_{i(n)}^{h(n)} y\right\|^{2} \leq & \left(1+\gamma_{h(n), i(n)}\right)\|x-y\|^{2} \\
& +\kappa_{i(n)}\left\|\left(I-T_{i(n)}^{h(n)}\right) x-\left(I-T_{i(n)}^{h(n)}\right) y\right\|^{2} \\
& +\tau_{h(n), i(n)}, \quad \forall x, y \in C,
\end{aligned}
$$


for all $n \in \mathbb{N} \cup\{0\}$ such that $n=(h-1) N+i$, where $i=i(n) \in\{0,1, \ldots, N-1\}, h=h(n) \geq 1$ is a positive integer and $h(n) \rightarrow \infty$ as $n \rightarrow \infty$.

Subsequently, Hu and Cai [7] modified Ishikawa's iteration method (see [45]) for finding a common element of the set of common fixed points for a finite family of asymptotically $\kappa_{i}$-SPC mappings in the intermediate sense and the set of solutions of the equilibrium problems (see also Duan and Zhao [8]) as the following theorem.

Theorem HC Let $C$ be a nonempty closed convex subset of a real Hilbert space $H, \Phi$ : $C \times C \rightarrow \mathbb{R}$ be a bifunction satisfying the conditions (A1)-(A4), and let $A: C \rightarrow H$ be a $\xi$-cocoercive mapping. Let $N \geq 1$ be an integer. For each $i=0,1, \ldots, N-1$, let $\left\{T_{i}: C \rightarrow C\right\}$ be a finite family of uniformly continuous and asymptotically $\kappa_{i}$-SPC mappings in the intermediate sense defined as in (1.17) when $\kappa_{i} \in[0,1)$ with the sequences $\left\{\gamma_{n, i}\right\},\left\{\tau_{n, i}\right\} \subset[0, \infty)$ such that $\lim _{n \rightarrow \infty} \gamma_{n, i}=\lim _{n \rightarrow \infty} \tau_{n, i}=0$. Let $\kappa=\max \left\{\kappa_{i}: 0 \leq i \leq N-1\right\}, \gamma_{n}=\max \left\{\gamma_{n, i}: 0 \leq\right.$ $i \leq N-1\}$ and $\tau_{n}=\max \left\{\tau_{n, i}: 0 \leq i \leq N-1\right\}$. Assume that $\Omega:=\bigcap_{i=0}^{N-1} F\left(T_{i}\right) \cap \operatorname{EP}(\Phi)$ is a nonempty bounded subset of $C$. For $x_{0}=x \in C$ chosen arbitrarily, suppose that $\left\{x_{n}\right\}$ and $\left\{u_{n}\right\}$ are generated iteratively by

$$
\left\{\begin{aligned}
u_{n} \in & C \text { such that } \\
& \Phi\left(u_{n}, y\right)+\left\langle A x_{n}, y-u_{n}\right\rangle+\frac{1}{r_{n}}\left\langle y-u_{n}, u_{n}-x_{n}\right\rangle \geq 0, \quad \forall y \in C, \\
z_{n}= & \left(1-\beta_{n}\right) u_{n}+\beta_{n} T_{i(n)}^{h(n)} u_{n}, \\
y_{n}= & \left(1-\alpha_{n}\right) u_{n}+\alpha_{n} z_{n}, \\
C_{n}= & \left\{z \in H:\left\|y_{n}-z\right\|^{2} \leq\left\|x_{n}-z\right\|^{2}+\theta_{n}\right\}, \\
Q_{n}= & \left\{z \in C:\left\langle x_{n}-z, x_{0}-x_{n}\right\rangle \geq 0\right\}, \\
x_{n+1}= & P_{C_{n} \cap Q_{n}}\left(x_{0}\right), \quad \forall n \in \mathbb{N} \cup\{0\},
\end{aligned}\right.
$$

where $\theta_{n}=\gamma_{h(n)} \cdot \Delta_{n}^{2}+\tau_{h(n)} \rightarrow 0$ as $n \rightarrow \infty$ such that $\Delta_{n}=\sup \left\{\left\|x_{n}-z\right\|: z \in \Omega\right\}<\infty$. If the control sequences $\left\{\alpha_{n}\right\},\left\{\beta_{n}\right\} \subset(0,1]$, and $\left\{r_{n}\right\} \subset[a, b]$ are chosen such that $0<\alpha \leq \alpha_{n} \leq 1$, $0<\beta \leq \beta_{n} \leq 1-\kappa$, and $0<a \leq r_{n} \leq b<2 \xi$, then the sequences $\left\{x_{n}\right\}$ and $\left\{u_{n}\right\}$ converge strongly to $P_{\Omega}\left(x_{0}\right)$.

In this paper, we study the sequences $\left\{x_{n, i}\right\}_{n=1}^{\infty}$ generated by modifying Mann's iteration method (1.10) for an infinite family of asymptotically $\kappa_{i}$-SPC mappings in the intermediate sense. For each $i=1,2, \ldots$, let $\left\{T_{i}: C \rightarrow C\right\}$ be an infinite family of asymptotically $\kappa_{i}$-SPC mappings in the intermediate sense with the sequences $\left\{\gamma_{n, i}\right\}_{n=1}^{\infty},\left\{\tau_{n, i}\right\}_{n=1}^{\infty} \subset[0, \infty)$ such that $\lim _{n \rightarrow \infty} \gamma_{n, i}=\lim _{n \rightarrow \infty} \tau_{n, i}=0$. One has

$$
\begin{aligned}
\left\|T_{i}^{n} x-T_{i}^{n} y\right\|^{2} \leq & \left(1+\gamma_{n, i}\right)\|x-y\|^{2} \\
& +\kappa_{i}\left\|\left(I-T_{i}^{n}\right) x-\left(I-T_{i}^{n}\right) y\right\|^{2}+\tau_{n, i}, \quad \forall x, y \in C,
\end{aligned}
$$

for all $n \in \mathbb{N}$. For each $i=1,2, \ldots$, let $x_{1, i} \in C$ and $\left\{\alpha_{n, i}\right\}_{n=1}^{\infty}$ be a sequence in $[0,1]$, and let the sequences $\left\{x_{n, i}\right\}_{n=1}^{\infty}$ be generated in the following way:

$$
x_{n+1, i}=\left(1-\alpha_{n, i}\right) x_{n, i}+\alpha_{n, i} T_{i}^{n} x_{n, i}, \quad \forall n \in \mathbb{N} .
$$


Quite recently, Ezeora and Shehu [9] introduced an iterative scheme for finding a common fixed point of an infinite family of asymptotically $\kappa_{i}$-SPC mappings in the intermediate sense as the following theorem.

Theorem ES Let $C$ be a nonempty closed convex subset of a real Hilbert space $H$. For each $i=1,2, \ldots$, let $\left\{T_{i}: C \rightarrow C\right\}$ be an infinite family of uniformly continuous and asymptotically $\kappa_{i}$-SPC mappings in the intermediate sense defined as in $(1.18)$ when $\kappa_{i} \in[0,1)$ with the sequences $\left\{\gamma_{n, i}\right\}_{n=1}^{\infty},\left\{\tau_{n, i}\right\}_{n=1}^{\infty} \subset[0, \infty)$ such that $\lim _{n \rightarrow \infty} \gamma_{n, i}=\lim _{n \rightarrow \infty} \tau_{n, i}=0$. Assume that $\Omega:=\bigcap_{i=1}^{\infty} F\left(T_{i}\right)$ is a nonempty bounded subset of C. For $x_{1}=x \in C$ chosen arbitrarily, suppose that $\left\{x_{n}\right\}_{n=1}^{\infty}$ is generated iteratively by

$$
\left\{\begin{array}{l}
y_{n, i}=\left(1-\alpha_{n, i}\right) x_{n}+\alpha_{n, i} T_{i}^{n} x_{n}, \quad n \geq 1, \\
C_{n, i}=\left\{z \in C:\left\|y_{n, i}-z\right\|^{2} \leq\left\|x_{n}-z\right\|^{2}+\theta_{n, i}\right\}, \\
C_{n}=\bigcap_{i=1}^{\infty} C_{n, i}, \\
Q_{n}=\left\{z \in Q_{n-1}:\left\langle x_{n}-z, x_{1}-x_{n}\right\rangle \geq 0\right\}, \quad n \geq 2, \\
Q_{1}=C, \\
x_{n+1}=P_{C_{n} \cap Q_{n}}\left(x_{1}\right), \quad \forall n \in \mathbb{N},
\end{array}\right.
$$

where $\theta_{n, i}=\gamma_{n, i} \cdot \Delta_{n}^{2}+\tau_{n, i}(i=1,2, \ldots), \Delta_{n}=\sup \left\{\left\|x_{n}-z\right\|: z \in \Omega\right\}<\infty$ and $\left\{\alpha_{n, i}\right\}_{n=1}^{\infty} \subset(0,1]$ $(i=1,2, \ldots)$ such that $0<\alpha_{i} \leq \alpha_{n, i} \leq 1-\kappa_{i}$. Then the sequence $\left\{x_{n}\right\}_{n=1}^{\infty}$ converges strongly to $P_{\Omega}\left(x_{1}\right)$.

Inspired and motivated by the works mentioned above, in this paper, we introduce a new iterative scheme (3.1) below by modifying Mann's iteration method (1.10) to find a common element for the set of common fixed points of an infinite family of asymptotically $\kappa_{i}$-SPC mappings in the intermediate sense, the set of solutions of the cocoercive quasivariational inclusions problems, and the set of solutions of the mixed equilibrium problems in Hilbert spaces. The strong convergence theorem of the iterative scheme to a common element of the three aforementioned sets is obtained based on the shrinking projection method which extends and improves that of Ezeora and Shehu [9] and many others.

\section{Preliminaries}

We collect the following lemmas which be used in the proof of the main results in the next section.

Lemma 2.1 (see [1]) Let C be a nonempty closed convex subset of a Hilbert space H. Then the following inequality holds:

$$
\left\langle x-P_{C} x, P_{C} x-y\right\rangle \geq 0, \quad \forall x \in H, y \in C .
$$

Lemma 2.2 (see [46]) Let $H$ be a Hilbert space. For all $x, y, z \in H$ and $\alpha, \beta, \gamma \in[0,1]$ such that $\alpha+\beta+\gamma=1$, one has

$$
\|\alpha x+\beta y+\gamma z\|^{2}=\alpha\|x\|^{2}+\beta\|y\|^{2}+\gamma\|z\|^{2}-\alpha \beta\|x-y\|^{2}-\alpha \gamma\|x-z\|^{2}-\beta \gamma\|y-z\|^{2} .
$$


Lemma 2.3 (see [25]) Let $C$ be a nonempty closed convex subset of a real Hilbert space $H$, $\Phi: C \times C \rightarrow \mathbb{R}$ satisfying the conditions (A1)-(A5), and let $\varphi: C \rightarrow \mathbb{R} \cup\{+\infty\}$ be a proper lower semicontinuous and convex function. Assume that either (B1) or (B2) holds. For $r>0$, define a mapping $S_{r}: C \rightarrow C$ as follows:

$$
S_{r}(x)=\left\{z \in C: \Phi(z, y)+\varphi(y)-\varphi(z)+\frac{1}{r}\langle y-z, z-x\rangle \geq 0, \forall y \in C\right\}
$$

for all $x \in C$. Then the following statements hold:

(1) for each $x \in C, S_{r}(x) \neq \emptyset$;

(2) $S_{r}$ is single-valued;

(3) $S_{r}$ is firmly nonexpansive; that is, for any $x, y \in C$,

$$
\left\|S_{r} x-S_{r} y\right\|^{2} \leq\left\langle S_{r} x-S_{r} y, x-y\right\rangle
$$

(4) $F\left(S_{r}\right)=\operatorname{MEP}(\Phi, \varphi)$;

(5) $\operatorname{MEP}(\Phi, \varphi)$ is closed and convex.

Lemma 2.4 (see [42]) Let $M: H \rightarrow 2^{H}$ be a maximal monotone mapping and let $B: H \rightarrow$ $H$ be an $\alpha$-inverse-strongly monotone mapping. Then, the following statements hold:

(1) $B$ is a $(1 / \alpha)$-Lipschitz continuous and monotone mapping.

(2) $u \in H$ is a solution of quasivariational inclusion (1.13) if and only if $u=J_{M, \lambda}(u-\lambda B u)$, for all $\lambda>0$, that is,

$$
V I(H, B, M)=F\left(J_{M, \lambda}(I-\lambda B)\right), \quad \forall \lambda>0 .
$$

(3) If $\lambda \in(0,2 \alpha]$, then $V I(H, B, M)$ is a closed convex subset in $H$, and the mapping $I-\lambda B$ is nonexpansive, where $I$ is the identity mapping on $H$.

(4) The resolvent operator $J_{M, \lambda}$ associated with $M$ is single-valued and nonexpansive for all $\lambda>0$.

(5) The resolvent operator $J_{M, \lambda}$ is 1-inverse-strongly monotone; that is,

$$
\left\|J_{M, \lambda}(x)-J_{M, \lambda}(y)\right\|^{2} \leq\left\langle x-y, J_{M, \lambda}(x)-J_{M, \lambda}(y)\right\rangle, \quad \forall x, y \in H .
$$

Lemma 2.5 (see [47]) Let $M: H \rightarrow 2^{H}$ be a maximal monotone mapping and let $B: H \rightarrow$ $H$ be a Lipschitz continuous mapping. Then the mapping $S=M+B: H \rightarrow 2^{H}$ is a maximal monotone mapping.

Lemma 2.6 (see [6]) Let $C$ be a nonempty closed convex subset of a real Hilbert space $H$ and let $T: C \rightarrow C$ be a uniformly continuous and asymptotically $\kappa$-strictly pseudocontractive mapping in the intermediate sense defined as in $(1.9)$ when $\kappa \in[0,1)$ with the sequences $\left\{\gamma_{n}\right\},\left\{\tau_{n}\right\} \subset[0, \infty)$ such that $\lim _{n \rightarrow \infty} \gamma_{n}=\lim _{n \rightarrow \infty} \tau_{n}=0$. Then the following statements hold:

(1) $\left\|T^{n} x-T^{n} y\right\| \leq \frac{1}{1-\kappa}\left(\kappa\|x-y\|+\sqrt{\left.\left(1+(1-\kappa) \gamma_{n}\right)\|x-y\|^{2}+(1-\kappa) \tau_{n}\right)}\right.$, for all $x, y \in C$ and $n \in \mathbb{N}$.

(2) If $\left\{x_{n}\right\}$ is a sequence in $C$ such that $\lim _{n \rightarrow \infty}\left\|x_{n}-x_{n+1}\right\|=0$ and $\lim _{n \rightarrow \infty}\left\|x_{n}-T^{n} x_{n}\right\|=0$. Then $\lim _{n \rightarrow \infty}\left\|x_{n}-T x_{n}\right\|=0$. 
(3) $I-T$ is demiclosed at zero in the sense that if $\left\{x_{n}\right\}$ is a sequence in $C$ such that $x_{n} \rightarrow x \in C$ as $n \rightarrow \infty$, and $\lim \sup _{m \rightarrow \infty} \limsup _{n \rightarrow \infty}\left\|x_{n}-T^{m} x_{n}\right\|=0$, then $(I-T) x=0$.

(4) $F(T)$ is closed and convex.

\section{Main results}

Theorem 3.1 Let $H$ be a real Hilbert space, $\Phi$ be a bifunction from $H \times H$ into $\mathbb{R}$ satisfying the conditions (A1)-(A5), and let $\varphi: H \rightarrow \mathbb{R} \cup\{+\infty\}$ be a proper lower semicontinuous and convex function with the assumption that either (B1) or (B2) holds. Let $M: H \rightarrow 2^{H}$ be a maximal monotone mapping and let $B: H \rightarrow H$ be a $\xi$-cocoercive mapping. For each $i=$ $1,2, \ldots$, let $\left\{T_{i}: H \rightarrow H\right\}$ be an infinite family of uniformly continuous and asymptotically $\kappa_{i}$-SPC mappings in the intermediate sense defined as in (1.18) when $\kappa_{i} \in[0,1)$ with the sequences $\left\{\gamma_{n, i}\right\}_{n=1}^{\infty},\left\{\tau_{n, i}\right\}_{n=1}^{\infty} \subset[0, \infty)$ such that $\lim _{n \rightarrow \infty} \gamma_{n, i}=\lim _{n \rightarrow \infty} \tau_{n, i}=0$. Assume that $\Omega:=\bigcap_{i=1}^{\infty} F\left(T_{i}\right) \cap V I(H, B, M) \cap \operatorname{MEP}(\Phi, \varphi)$ is a nonempty bounded subset of $H$. For $x_{1}=$ $x \in H$ chosen arbitrarily, suppose that $\left\{x_{n}\right\}_{n=1}^{\infty}$ is generated iteratively by

$$
\left\{\begin{array}{l}
u_{n} \in H \text { such that } \\
\quad \Phi\left(u_{n}, y\right)+\varphi(y)-\varphi\left(u_{n}\right)+\frac{1}{r_{n}}\left\langle y-u_{n}, u_{n}-x_{n}\right\rangle \geq 0, \quad \forall y \in H, \\
y_{n, i}=\left(1-\alpha_{n, i}-\beta_{n, i}\right) u_{n}+\alpha_{n, i} T_{i}^{n} u_{n}+\beta_{n, i} J_{M, \lambda}\left(u_{n}-\lambda B u_{n}\right), \\
C_{n+1, i}=\left\{z \in C_{n} \cap Q_{n}:\left\|y_{n, i}-z\right\|^{2} \leq\left\|x_{n}-z\right\|^{2}+\theta_{n, i}\right\}, \\
C_{n+1}=\bigcap_{i=1}^{\infty} C_{n+1, i}, \\
Q_{n+1}=\left\{z \in C_{n} \cap Q_{n}:\left\langle x_{n}-z, x_{1}-x_{n}\right\rangle \geq 0\right\}, \\
C_{1, i}=C_{1}=Q_{1}=H, \\
x_{n+1}=P_{C_{n+1} \cap Q_{n+1}}\left(x_{1}\right), \quad \forall n \in \mathbb{N},
\end{array}\right.
$$

where $\theta_{n, i}=\gamma_{n, i} \cdot \Delta_{n}^{2}+\tau_{n, i}(i=1,2, \ldots)$ and $\Delta_{n}=\sup \left\{\left\|x_{n}-z\right\|: z \in \Omega\right\}<\infty$ satisfying the following conditions:

(C1) $\left\{\alpha_{n, i}\right\}_{n=1}^{\infty} \subset\left[a_{1, i}, b_{1, i}\right]$ and $\left\{\beta_{n, i}\right\}_{n=1}^{\infty} \subset\left[a_{2, i}, b_{2, i}\right](i=1,2, \ldots)$ such that $0<a_{j, i}<b_{j, i}<1$ for each $j=1,2$, and $b_{1, i}+b_{2, i}<1-\kappa_{i}(i=1,2, \ldots)$;

(C2) $\lambda \in(0,2 \xi]$ and $\left\{r_{n}\right\}_{n=1}^{\infty} \subset[r, \infty)$ for some $r>0$.

Then the sequence $\left\{x_{n}\right\}_{n=1}^{\infty}$ converges strongly to $w=P_{\Omega}\left(x_{1}\right)$.

Proof Pick $p \in \Omega$ and fix $i=1,2, \ldots$ From (3.1), by the definition of $S_{r_{n}}$ in Lemma 2.3, we have

$$
u_{n}=S_{r_{n}} x_{n} \in \operatorname{dom} \varphi
$$

and by $T_{i} p=p$, Lemmas 2.3(4) and 2.4(2), we have

$$
T_{i}^{n} p=S_{r_{n}} p=J_{M, \lambda}(I-\lambda B) p=p .
$$

From Lemma 2.3(3), we know that $S_{r_{n}}$ is nonexpansive. Therefore, by (3.2) and (3.3), we have

$$
\left\|u_{n}-p\right\|=\left\|S_{r_{n}} x_{n}-S_{r_{n}} p\right\| \leq\left\|x_{n}-p\right\| .
$$


Let $t_{n}=J_{M, \lambda}\left(u_{n}-\lambda B u_{n}\right)$. From Lemmas 2.4(3) and 2.4(4), we know that $J_{M, \lambda}$ and $I-\lambda B$ are nonexpansive. Therefore, by (3.3), we have

$$
\left\|t_{n}-p\right\|=\left\|J_{M, \lambda}\left(u_{n}-\lambda B u_{n}\right)-J_{M, \lambda}(p-\lambda B p)\right\| \leq\left\|u_{n}-p\right\| .
$$

By (3.3), (3.4), (3.5), Lemma 2.2, and the asymptotically $\kappa_{i}$-SPC in the intermediate sense of $T_{i}$, we have

$$
\begin{aligned}
\left\|y_{n, i}-p\right\|^{2}= & \left\|\left(1-\alpha_{n, i}-\beta_{n, i}\right)\left(u_{n}-p\right)+\alpha_{n, i}\left(T_{i}^{n} u_{n}-p\right)+\beta_{n, i}\left(t_{n}-p\right)\right\|^{2} \\
= & \left(1-\alpha_{n, i}-\beta_{n, i}\right)\left\|u_{n}-p\right\|^{2}+\alpha_{n, i}\left\|T_{i}^{n} u_{n}-p\right\|^{2}+\beta_{n, i}\left\|t_{n}-p\right\|^{2} \\
& -\alpha_{n, i}\left(1-\alpha_{n, i}-\beta_{n, i}\right)\left\|u_{n}-T_{i}^{n} u_{n}\right\|^{2} \\
& -\beta_{n, i}\left(1-\alpha_{n, i}-\beta_{n, i}\right)\left\|u_{n}-t_{n}\right\|^{2}-\alpha_{n, i} \beta_{n, i}\left\|T_{i}^{n} u_{n}-t_{n}\right\|^{2} \\
\leq & \left(1-\alpha_{n, i}-\beta_{n, i}\right)\left\|u_{n}-p\right\|^{2} \\
& +\alpha_{n, i}\left(\left(1+\gamma_{n, i}\right)\left\|u_{n}-p\right\|^{2}+\kappa_{i}\left\|u_{n}-T_{i}^{n} u_{n}\right\|^{2}+\tau_{n, i}\right) \\
& +\beta_{n, i}\left\|t_{n}-p\right\|^{2}-\alpha_{n, i}\left(1-\alpha_{n, i}-\beta_{n, i}\right)\left\|u_{n}-T_{i}^{n} u_{n}\right\|^{2} \\
& -\beta_{n, i}\left(1-\alpha_{n, i}-\beta_{n, i}\right)\left\|u_{n}-t_{n}\right\|^{2}-\alpha_{n, i} \beta_{n, i}\left\|T_{i}^{n} u_{n}-t_{n}\right\|^{2} \\
\leq & \left(1+\alpha_{n, i} \gamma_{n, i}\right)\left\|u_{n}-p\right\|^{2}+\alpha_{n, i} \tau_{n, i} \\
& -\alpha_{n, i}\left(1-\alpha_{n, i}-\beta_{n, i}-\kappa_{i}\right)\left\|u_{n}-T_{i}^{n} u_{n}\right\|^{2} \\
& -\beta_{n, i}\left(1-\alpha_{n, i}-\beta_{n, i}\right)\left\|u_{n}-t_{n}\right\|^{2}-\alpha_{n, i} \beta_{n, i}\left\|T_{i}^{n} u_{n}-t_{n}\right\|^{2} \\
\leq & \left(1+\gamma_{n, i}\right)\left\|u_{n}-p\right\|^{2}+\tau_{n, i}-\alpha_{n, i}\left(1-\alpha_{n, i}-\beta_{n, i}-\kappa_{i}\right)\left\|u_{n}-T_{i}^{n} u_{n}\right\|^{2} \\
\leq & \left\|x_{n}-p\right\|^{2}+\theta_{n, i},
\end{aligned}
$$

where $\theta_{n, i}:=\gamma_{n, i} \cdot \Delta_{n}^{2}+\tau_{n, i}$ and $\Delta_{n}:=\sup \left\{\left\|x_{n}-z\right\|: z \in \Omega\right\}$.

Firstly, we show that $C_{n} \cap Q_{n}$ is closed and convex for all $n \in \mathbb{N}$. It is obvious that $C_{1} \cap Q_{1}$ is closed and, by mathematical induction, that $C_{n} \cap Q_{n}$ is closed for all $n \geq 2$, that is, $C_{n} \cap Q_{n}$ is closed for all $n \in \mathbb{N}$. Since, for any $z \in H,\left\|y_{n, i}-z\right\|^{2} \leq\left\|x_{n}-z\right\|^{2}+\theta_{n, i}$ is equivalent to

$$
\left\|y_{n, i}-x_{n}\right\|^{2}+2\left\langle y_{n, i}-x_{n}, x_{n}-z\right\rangle-\theta_{n, i} \leq 0
$$

for all $n \in \mathbb{N}$. Therefore, for any $z_{1}, z_{2} \in C_{n+1} \cap Q_{n+1} \subset C_{n} \cap Q_{n}$ and $\epsilon \in(0,1)$, we have

$$
\begin{aligned}
&\left\|y_{n, i}-x_{n}\right\|^{2}+2\left\langle y_{n, i}-x_{n}, x_{n}-\left(\epsilon z_{1}+(1-\epsilon) z_{2}\right)\right\rangle-\theta_{n, i} \\
&= \epsilon\left(\left\|y_{n, i}-x_{n}\right\|^{2}+2\left\langle y_{n, i}-x_{n}, x_{n}-z_{1}\right\rangle-\theta_{n, i}\right) \\
& \quad+(1-\epsilon)\left(\left\|y_{n, i}-x_{n}\right\|^{2}+2\left\langle y_{n, i}-x_{n}, x_{n}-z_{2}\right\rangle-\theta_{n, i}\right) \\
& \leq 0, \\
&\left\langle x_{n}-\right.\left.\left(\epsilon z_{1}+(1-\epsilon) z_{2}\right), x_{1}-x_{n}\right\rangle \\
&= \epsilon\left\langle x_{n}-z_{1}, x_{1}-x_{n}\right\rangle+(1-\epsilon)\left\langle x_{n}-z_{2}, x_{1}-x_{n}\right\rangle \\
& \geq 0
\end{aligned}
$$


for all $n \in \mathbb{N}$. Since $C_{1} \cap Q_{1}$ is convex, by putting $n=1$ in (3.7), (3.8), and (3.9), we have that $C_{2} \cap Q_{2}$ is convex. Suppose that $x_{k}$ is given and $C_{k} \cap Q_{k}$ is convex for some $k \geq 2$. It follows by putting $n=k$ in (3.7), (3.8), and (3.9) that $C_{k+1} \cap Q_{k+1}$ is convex. Therefore, by mathematical induction, we have that $C_{n} \cap Q_{n}$ is convex for all $n \geq 2$, that is, $C_{n} \cap Q_{n}$ is convex for all $n \in \mathbb{N}$. Hence, we obtain that $C_{n} \cap Q_{n}$ is closed and convex for all $n \in \mathbb{N}$.

Next, we show that $\Omega \subset C_{n} \cap Q_{n}$ for all $n \in \mathbb{N}$. It is obvious that $p \in \Omega \subset H=C_{1} \cap Q_{1}$. Therefore, by (3.1) and (3.6), we have $p \in C_{2, i}$ for all $i$, and so $p \in C_{2}$, and note that $p \in H=Q_{2}$, and so $p \in C_{2} \cap Q_{2}$. Hence, we have $\Omega \subset C_{2} \cap Q_{2}$. Since $C_{2} \cap Q_{2}$ is a nonempty closed convex subset of $H$, there exists a unique element $x_{2} \in C_{2} \cap Q_{2}$ such that $x_{2}=P_{C_{2} \cap Q_{2}}\left(x_{1}\right)$. Suppose that $x_{k} \in C_{k} \cap Q_{k}$ is given such that $x_{k}=P_{C_{k} \cap Q_{k}}\left(x_{1}\right)$, and $p \in \Omega \subset C_{k} \cap Q_{k}$ for some $k \geq 2$. Therefore, by (3.1) and (3.6), we have $p \in C_{k+1, i}$ for all $i$, and so $p \in C_{k+1}$. Since $x_{k}=P_{C_{k} \cap Q_{k}}\left(x_{1}\right)$, therefore, by Lemma 2.1, we have

$$
\left\langle x_{k}-z, x_{1}-x_{k}\right\rangle \geq 0
$$

for all $z \in C_{k} \cap Q_{k}$. Thus, by (3.1), we have $p \in Q_{k+1}$, and so $p \in C_{k+1} \cap Q_{k+1}$. Hence, we have $\Omega \subset C_{k+1} \cap Q_{k+1}$. Since $C_{k+1} \cap Q_{k+1}$ is a nonempty closed convex subset of $H$, there exists a unique element $x_{k+1} \in C_{k+1} \cap Q_{k+1}$ such that $x_{k+1}=P_{C_{k+1} \cap Q_{k+1}}\left(x_{1}\right)$. Therefore, by mathematical induction, we obtain that $\Omega \subset C_{n} \cap Q_{n}$ for all $n \geq 2$, and so $\Omega \subset C_{n} \cap Q_{n}$ for all $n \in \mathbb{N}$, and we can define $x_{n+1}=P_{C_{n+1} \cap Q_{n+1}}\left(x_{1}\right)$ for all $n \in \mathbb{N}$. Hence, we obtain that the iteration (3.1) is well defined, and by Lemmas 2.3(5), 2.4(3), and 2.6(4), we also obtain that $P_{\Omega}\left(x_{1}\right)$ is well defined.

Next, we show that $\left\{x_{n}\right\}$ is bounded. Since $x_{n}=P_{C_{n} \cap Q_{n}}\left(x_{1}\right)$ for all $n \in \mathbb{N}$, we have

$$
\left\|x_{n}-x_{1}\right\| \leq\left\|z-x_{1}\right\|
$$

for all $z \in C_{n} \cap Q_{n}$. It follows by $p \in \Omega \subset C_{n} \cap Q_{n}$ that $\left\|x_{n}-x_{1}\right\| \leq\left\|p-x_{1}\right\|$ for all $n \in \mathbb{N}$. This implies that $\left\{x_{n}\right\}$ is bounded, and so are $\left\{u_{n}\right\},\left\{t_{n}\right\}$, and $\left\{y_{n, i}\right\}$ for each $i=1,2, \ldots$.

Next, we show that $\left\|x_{n}-x_{n+1}\right\| \rightarrow 0$ as $n \rightarrow \infty$. Since $x_{n+1}=P_{C_{n+1} \cap Q_{n+1}}\left(x_{1}\right) \in C_{n+1} \cap$ $Q_{n+1} \subset C_{n} \cap Q_{n}$, therefore, by (3.10), we have $\left\|x_{n}-x_{1}\right\| \leq\left\|x_{n+1}-x_{1}\right\|$ for all $n \in \mathbb{N}$. This implies that $\left\{\left\|x_{n}-x_{1}\right\|\right\}$ is a bounded nondecreasing sequence, there exists the limit of $\left\|x_{n}-x_{1}\right\|$, that is,

$$
\lim _{n \rightarrow \infty}\left\|x_{n}-x_{1}\right\|=m_{1}
$$

for some $m_{1} \geq 0$. Since $x_{n+1} \in Q_{n+1}$, therefore, by (3.1), we have

$$
\left\langle x_{n}-x_{n+1}, x_{1}-x_{n}\right\rangle \geq 0
$$

It follows that

$$
\begin{aligned}
\left\|x_{n}-x_{n+1}\right\|^{2} & =\left\|x_{n}-x_{1}\right\|^{2}+2\left\langle x_{n}-x_{1}, x_{1}-x_{n}\right\rangle+2\left\langle x_{n}-x_{1}, x_{n}-x_{n+1}\right\rangle+\left\|x_{n+1}-x_{1}\right\|^{2} \\
& \leq\left\|x_{n+1}-x_{1}\right\|^{2}-\left\|x_{n}-x_{1}\right\|^{2} .
\end{aligned}
$$

Therefore, by (3.11), we obtain

$$
\left\|x_{n}-x_{n+1}\right\| \rightarrow 0 \quad \text { as } n \rightarrow \infty .
$$


Next, we show that $\left\{x_{n}\right\}$ is a Cauchy sequence. Observe that

$$
C_{1} \cap Q_{1} \supset C_{2} \cap Q_{2} \supset \cdots \supset C_{n} \cap Q_{n} \supset \cdots \supset \Omega .
$$

It follows that

$$
x_{n+m}=P_{C_{n+m} \cap Q_{n+m}}\left(x_{1}\right) \in C_{n+m} \cap Q_{n+m} \subset C_{n+1} \cap Q_{n+1} \subset Q_{n+1}
$$

for each $m \geq 1$. Therefore, by (3.1), we have

$$
\left\langle x_{n}-x_{n+m}, x_{1}-x_{n}\right\rangle \geq 0 .
$$

Thus, we have

$$
\begin{aligned}
\left\|x_{n+m}-x_{n}\right\|^{2} & =\left\|x_{n+m}-x_{1}\right\|^{2}+\left\|x_{n}-x_{1}\right\|^{2}-2\left\langle x_{n+m}-x_{1}, x_{n}-x_{1}\right\rangle \\
& =\left\|x_{n+m}-x_{1}\right\|^{2}-\left\|x_{n}-x_{1}\right\|^{2}-2\left\langle x_{n+m}-x_{n}, x_{n}-x_{1}\right\rangle \\
& \leq\left\|x_{n+m}-x_{1}\right\|^{2}-\left\|x_{n}-x_{1}\right\|^{2} .
\end{aligned}
$$

Hence, by (3.11), we obtain that $\left\|x_{n+m}-x_{n}\right\| \rightarrow 0$ as $n \rightarrow \infty$, which implies that $\left\{x_{n}\right\}$ is a Cauchy sequence in $H$, and then there exists a point $w \in H$ such that $x_{n} \rightarrow w$ as $n \rightarrow \infty$.

Next, we show that $\left\|y_{n, i}-x_{n}\right\| \rightarrow 0$ as $n \rightarrow \infty$. From (3.1), we have $x_{n+1}=P_{C_{n+1} \cap Q_{n+1}}\left(x_{1}\right) \in$ $C_{n+1} \cap Q_{n+1} \subset C_{n+1} \subset C_{n+1, i}$. Therefore, we have

$$
\left\|y_{n, i}-x_{n+1}\right\|^{2} \leq\left\|x_{n}-x_{n+1}\right\|^{2}+\theta_{n, i}
$$

It follows by (3.13) and $\lim _{n \rightarrow \infty} \theta_{n, i}=0$ that

$$
\left\|y_{n, i}-x_{n+1}\right\| \rightarrow 0 \quad \text { as } n \rightarrow \infty
$$

Since,

$$
\left\|y_{n, i}-x_{n}\right\| \leq\left\|y_{n, i}-x_{n+1}\right\|+\left\|x_{n+1}-x_{n}\right\|
$$

Therefore, by (3.13) and (3.17), we obtain

$$
\left\|y_{n, i}-x_{n}\right\| \rightarrow 0 \quad \text { as } n \rightarrow \infty
$$

Next, we show that $\left\|u_{n}-x_{n}\right\| \rightarrow 0$ and $\left\|u_{n+1}-u_{n}\right\| \rightarrow 0$ as $n \rightarrow \infty$. By (3.2), (3.3), and the firmly nonexpansiveness of $S_{r_{n}}$ in Lemma 2.3(3), we have

$$
\begin{aligned}
\left\|u_{n}-p\right\|^{2} & \leq\left\langle S_{r_{n}} x_{n}-S_{r_{n}} p, x_{n}-p\right\rangle=\left\langle u_{n}-p, x_{n}-p\right\rangle \\
& =\frac{1}{2}\left(\left\|u_{n}-p\right\|^{2}+\left\|x_{n}-p\right\|^{2}-\left\|u_{n}-x_{n}\right\|^{2}\right),
\end{aligned}
$$

which implies that

$$
\left\|u_{n}-p\right\|^{2} \leq\left\|x_{n}-p\right\|^{2}-\left\|u_{n}-x_{n}\right\|^{2} .
$$


By (3.4), (3.6), and (3.20), we have

$$
\begin{aligned}
\left\|y_{n, i}-p\right\|^{2} & \leq\left(1+\gamma_{n, i}\right)\left\|u_{n}-p\right\|^{2}+\tau_{n, i} \leq\left\|u_{n}-p\right\|^{2}+\theta_{n, i} \\
& \leq\left\|x_{n}-p\right\|^{2}-\left\|u_{n}-x_{n}\right\|^{2}+\theta_{n, i},
\end{aligned}
$$

which implies that

$$
\begin{aligned}
\left\|u_{n}-x_{n}\right\|^{2} & \leq\left\|x_{n}-p\right\|^{2}-\left\|y_{n, i}-p\right\|^{2}+\theta_{n, i} \\
& \leq\left\|x_{n}-y_{n, i}\right\|\left(\left\|x_{n}-p\right\|+\left\|y_{n, i}-p\right\|\right)+\theta_{n, i} .
\end{aligned}
$$

Therefore, by (3.19) and $\lim _{n \rightarrow \infty} \theta_{n, i}=0$, we obtain

$$
\left\|u_{n}-x_{n}\right\| \rightarrow 0 \quad \text { as } n \rightarrow \infty
$$

Since

$$
\left\|u_{n+1}-u_{n}\right\| \leq\left\|u_{n+1}-x_{n+1}\right\|+\left\|x_{n+1}-x_{n}\right\|+\left\|x_{n}-u_{n}\right\|,
$$

therefore, by (3.13) and (3.22), we obtain

$$
\left\|u_{n+1}-u_{n}\right\| \rightarrow 0 \quad \text { as } n \rightarrow \infty \text {. }
$$

Next, we show that $w \in \bigcap_{i=1}^{\infty} F\left(T_{i}\right)$. From (3.4) and (3.6), we have

$$
\begin{aligned}
\left\|y_{n, i}-p\right\|^{2} & \leq\left(1+\gamma_{n, i}\right)\left\|u_{n}-p\right\|^{2}+\tau_{n, i}-\alpha_{n, i}\left(1-\alpha_{n, i}-\beta_{n, i}-\kappa_{i}\right)\left\|u_{n}-T_{i}^{n} u_{n}\right\|^{2} \\
& \leq\left\|u_{n}-p\right\|^{2}+\theta_{n, i}-\alpha_{n, i}\left(1-\alpha_{n, i}-\beta_{n, i}-\kappa_{i}\right)\left\|u_{n}-T_{i}^{n} u_{n}\right\|^{2}
\end{aligned}
$$

which implies that

$$
\begin{aligned}
& a_{1, i}\left(1-b_{1, i}-b_{2, i}-\kappa_{i}\right)\left\|u_{n}-T_{i}^{n} u_{n}\right\|^{2} \\
& \quad \leq \alpha_{n, i}\left(1-\alpha_{n, i}-\beta_{n, i}-\kappa_{i}\right)\left\|u_{n}-T_{i}^{n} u_{n}\right\|^{2} \\
& \quad \leq\left\|u_{n}-p\right\|^{2}-\left\|y_{n, i}-p\right\|^{2}+\theta_{n, i} \\
& \quad \leq\left\|u_{n}-y_{n, i}\right\|\left(\left\|u_{n}-p\right\|+\left\|y_{n, i}-p\right\|\right)+\theta_{n, i} \\
& \quad \leq\left(\left\|u_{n}-x_{n}\right\|+\left\|x_{n}-y_{n, i}\right\|\right)\left(\left\|u_{n}-p\right\|+\left\|y_{n, i}-p\right\|\right)+\theta_{n, i} .
\end{aligned}
$$

Therefore, by (3.19), (3.22), and $\lim _{n \rightarrow \infty} \theta_{n, i}=0$, we obtain

$$
\left\|u_{n}-T_{i}^{n} u_{n}\right\| \rightarrow 0 \quad \text { as } n \rightarrow \infty .
$$

From (3.24) and (3.26), by Lemma 2.6(2), we have

$$
\left\|u_{n}-T_{i} u_{n}\right\| \rightarrow 0 \quad \text { as } n \rightarrow \infty
$$


Therefore, by (3.27) and the uniform continuity of $T_{i}$, it is easy to see, by mathematical induction on $m \in \mathbb{N}$, that

$$
\left\|T_{i}^{m} u_{n}-T_{i}^{m+1} u_{n}\right\| \rightarrow 0 \quad \text { as } n \rightarrow \infty
$$

for each $m \in \mathbb{N}$. Since, for any $m \in \mathbb{N}$, we have

$$
\left\|u_{n}-T_{i}^{m} u_{n}\right\| \leq\left\|u_{n}-T_{i} u_{n}\right\|+\left\|T_{i} u_{n}-T_{i}^{2} u_{n}\right\|+\cdots+\left\|T_{i}^{m-1} u_{n}-T_{i}^{m} u_{n}\right\|
$$

such that $T_{i}^{0}=I$, where $I$ is the identity mapping on $H$. Therefore, by (3.27) and (3.28), we obtain

$$
\left\|u_{n}-T_{i}^{m} u_{n}\right\| \rightarrow 0 \quad \text { as } n \rightarrow \infty
$$

From (3.22) and $x_{n} \rightarrow w$, we have $u_{n} \rightarrow w$ as $n \rightarrow \infty$. Therefore, from (3.29), by Lemma 2.6(3), we obtain that $w \in F\left(T_{i}\right)$ for all $i=1,2, \ldots$; that is, $w \in \bigcap_{i=1}^{\infty} F\left(T_{i}\right)$.

Next, we show that $w \in \operatorname{MEP}(\Phi, \varphi)$. From (3.1), we have

$$
0 \leq \Phi\left(u_{n}, y\right)+\varphi(y)-\varphi\left(u_{n}\right)+\frac{1}{r_{n}}\left\langle y-u_{n}, u_{n}-x_{n}\right\rangle, \quad \forall y \in H .
$$

It follows by the condition (A2) that

$$
\begin{aligned}
\Phi\left(y, u_{n}\right) & \leq \Phi\left(y, u_{n}\right)+\Phi\left(u_{n}, y\right)+\varphi(y)-\varphi\left(u_{n}\right)+\frac{1}{r_{n}}\left\langle y-u_{n}, u_{n}-x_{n}\right\rangle, \quad \forall y \in H \\
& \leq \varphi(y)-\varphi\left(u_{n}\right)+\frac{1}{r_{n}}\left\langle y-u_{n}, u_{n}-x_{n}\right\rangle, \quad \forall y \in H .
\end{aligned}
$$

Hence,

$$
\varphi(y)-\varphi\left(u_{n}\right)+\left\langle y-u_{n}, \frac{u_{n}-x_{n}}{r_{n}}\right\rangle \geq \Phi\left(y, u_{n}\right), \quad \forall y \in H .
$$

From (3.22) and $x_{n} \rightarrow w$, we have $u_{n} \rightarrow w$ as $n \rightarrow \infty$. Therefore, we obtain

$$
\Phi(y, w)+\varphi(w)-\varphi(y) \leq 0, \quad \forall y \in H .
$$

For a constant $t$ with $0<t<1$ and $y \in H$, let $y_{t}=t y+(1-t) w$. Since $y, w \in H$, thus, $y_{t} \in H$. So, from (3.31), we have

$$
\Phi\left(y_{t}, w\right)+\varphi(w)-\varphi\left(y_{t}\right) \leq 0 .
$$

By (3.32), the conditions (A1) and (A4), and the convexity of $\varphi$, we have

$$
\begin{aligned}
0 & =\Phi\left(y_{t}, y_{t}\right)+\varphi\left(y_{t}\right)-\varphi\left(y_{t}\right) \\
& \leq\left(t \Phi\left(y_{t}, y\right)+(1-t) \Phi\left(y_{t}, w\right)\right)+(t \varphi(y)+(1-t) \varphi(w))-\varphi\left(y_{t}\right) \\
& =t\left(\Phi\left(y_{t}, y\right)+\varphi(y)-\varphi\left(y_{t}\right)\right)+(1-t)\left(\Phi\left(y_{t}, w\right)+\varphi(w)-\varphi\left(y_{t}\right)\right) \\
& \leq t\left(\Phi\left(y_{t}, y\right)+\varphi(y)-\varphi\left(y_{t}\right)\right),
\end{aligned}
$$


which implies that

$$
\Phi\left(y_{t}, y\right)+\varphi(y)-\varphi\left(y_{t}\right) \geq 0
$$

Therefore, by the condition (A3) and the weakly lower semicontinuity of $\varphi$, we have $\Phi(w, y)+\varphi(y)-\varphi(w) \geq 0$ as $t \rightarrow 0$ for all $y \in H$, and hence we obtain that $w \in \operatorname{MEP}(\Phi, \varphi)$.

Next, we show that $w \in V I(H, B, M)$. From (3.1), we have

$$
y_{n, i}=\left(1-\alpha_{n, i}-\beta_{n, i}\right) u_{n}+\alpha_{n, i} T_{i}^{n} u_{n}+\beta_{n, i} t_{n},
$$

which implies that

$$
\begin{aligned}
a_{2, i}\left\|u_{n}-t_{n}\right\| & \leq \beta_{n, i}\left\|u_{n}-t_{n}\right\| \leq\left\|u_{n}-y_{n, i}\right\|+\alpha_{n, i}\left\|u_{n}-T_{i}^{n} u_{n}\right\| \\
& \leq\left\|u_{n}-x_{n}\right\|+\left\|x_{n}-y_{n, i}\right\|+\alpha_{n, i}\left\|u_{n}-T_{i}^{n} u_{n}\right\| .
\end{aligned}
$$

Therefore, by (3.19), (3.22), and (3.26), we obtain

$$
\left\|u_{n}-t_{n}\right\| \rightarrow 0 \quad \text { as } n \rightarrow \infty
$$

From Lemma 2.4(1), we have that $B$ is $(1 / \xi)$-Lipschitz continuous. Therefore, by Lemma 2.5 , we have that $M+B$ is maximal monotone. Let $(y, g) \in G(M+B)$, that is,

$$
g-B y \in M y \text {. }
$$

Since $t_{n}=J_{M, \lambda}\left(u_{n}-\lambda B u_{n}\right)$, we have $u_{n}-\lambda B u_{n} \in(I+\lambda M) t_{n}$. Therefore,

$$
\frac{1}{\lambda}\left(u_{n}-t_{n}-\lambda B u_{n}\right) \in M t_{n} .
$$

By (3.34), (3.35), and $M$ is maximal monotone, we have

$$
\begin{aligned}
0 & \leq\left\langle y-t_{n}, M y-M t_{n}\right\rangle \\
& =\left\langle y-t_{n}, g-B y-\frac{1}{\lambda}\left(u_{n}-t_{n}-\lambda B u_{n}\right)\right\rangle .
\end{aligned}
$$

It follows by the monotonicity of $B$ that

$$
\begin{aligned}
\left\langle y-t_{n}, g\right\rangle & \geq\left\langle y-t_{n}, B y+\frac{1}{\lambda}\left(u_{n}-t_{n}-\lambda B u_{n}\right)\right\rangle \\
& =\left\langle y-t_{n},\left(B y-B t_{n}\right)+\left(B t_{n}-B u_{n}\right)+\frac{1}{\lambda}\left(u_{n}-t_{n}\right)\right\rangle \\
& \geq\left\langle y-t_{n}, B t_{n}-B u_{n}\right\rangle+\frac{1}{\lambda}\left\langle y-t_{n}, u_{n}-t_{n}\right\rangle .
\end{aligned}
$$

From (3.33), we have $\left\|B t_{n}-B u_{n}\right\| \rightarrow 0$, and since $x_{n} \rightarrow w$, by (3.22) and (3.33), we have $t_{n} \rightarrow w$ as $n \rightarrow \infty$. Therefore, by (3.33) and (3.36), we obtain that $\langle y-w, g\rangle \geq 0$ as $n \rightarrow \infty$. It follows from the maximal monotonicity of $M+B$ that $0 \in(M+B) w$; that is, $w \in V I(H, B, M)$, and so $w \in \Omega$. 
Finally, we show that $w=P_{\Omega}\left(x_{1}\right)$. Since $\Omega \subset C_{n+1} \cap Q_{n+1} \subset Q_{n+1}$. Therefore, by (3.1), we have

$$
\left\langle x_{n}-z, x_{1}-x_{n}\right\rangle \geq 0, \quad \forall z \in \Omega
$$

It follows by $x_{n} \rightarrow w$ as $n \rightarrow \infty$ that

$$
\left\langle w-z, x_{1}-w\right\rangle \geq 0, \quad \forall z \in \Omega .
$$

Therefore, by Lemma 2.1, we obtain that $w=P_{\Omega}\left(x_{1}\right)$. This completes the proof.

Remark 3.2 The iteration (3.1) is different from the iterative scheme of Ezeora and Shehu [9] as follows:

1. The sequence $\left\{x_{n}\right\}$ is a projection sequence of $x_{1}$ onto $C_{n} \cap Q_{n}$ for all $n \in \mathbb{N}$ such that

$$
C_{1} \cap Q_{1} \supset C_{2} \cap Q_{2} \supset \cdots \supset C_{n} \cap Q_{n} \supset \cdots \supset \Omega .
$$

2. The proof to the strong convergence of the sequence $\left\{x_{n}\right\}$ is simple by a Cauchy sequence.

3. An approximate solution to a common element for the set of common fixed points of an infinite family of asymptotically strictly pseudocontractive mappings in the intermediate sense, the set of solutions of the cocoercive quasivariational inclusions problems, and the set of solutions of the mixed equilibrium problems by iteration is obtained.

We define the condition (B3) as the condition (B1) such that $\varphi=0$. If $\varphi=0$, then Theorem 3.1 is reduced immediately to the following result.

Corollary 3.3 Let $H$ be a real Hilbert space and let $\Phi$ be a bifunction from $H \times H$ into $\mathbb{R}$ satisfying the conditions (A1)-(A5) with the assumption that either (B2) or (B3) holds. Let $M: H \rightarrow 2^{H}$ be a maximal monotone mapping and let $B: H \rightarrow H$ be a $\xi$-cocoercive mapping. For each $i=1,2, \ldots$, let $\left\{T_{i}: H \rightarrow H\right\}$ be an infinite family of uniformly continuous and asymptotically $\kappa_{i}$-SPC mappings in the intermediate sense defined as in (1.18) when $\kappa_{i} \in$ $[0,1)$ with the sequences $\left\{\gamma_{n, i}\right\}_{n=1}^{\infty},\left\{\tau_{n, i}\right\}_{n=1}^{\infty} \subset[0, \infty)$ such that $\lim _{n \rightarrow \infty} \gamma_{n, i}=\lim _{n \rightarrow \infty} \tau_{n, i}=0$. Assume that $\Omega:=\bigcap_{i=1}^{\infty} F\left(T_{i}\right) \cap V I(H, B, M) \cap \mathrm{EP}(\Phi)$ is a nonempty bounded subset of $H$. For $x_{1}=x \in H$ chosen arbitrarily, suppose that $\left\{x_{n}\right\}_{n=1}^{\infty}$ is generated iteratively by

$$
\left\{\begin{array}{l}
u_{n} \in H \text { such that } \Phi\left(u_{n}, y\right)+\frac{1}{r_{n}}\left\langle y-u_{n}, u_{n}-x_{n}\right\rangle \geq 0, \quad \forall y \in H, \\
y_{n, i}=\left(1-\alpha_{n, i}-\beta_{n, i}\right) u_{n}+\alpha_{n, i} T_{i}^{n} u_{n}+\beta_{n, i} J_{M, \lambda}\left(u_{n}-\lambda B u_{n}\right), \\
C_{n+1, i}=\left\{z \in C_{n} \cap Q_{n}:\left\|y_{n, i}-z\right\|^{2} \leq\left\|x_{n}-z\right\|^{2}+\theta_{n, i}\right\}, \\
C_{n+1}=\bigcap_{i=1}^{\infty} C_{n+1, i}, \\
Q_{n+1}=\left\{z \in C_{n} \cap Q_{n}:\left\langle x_{n}-z, x_{1}-x_{n}\right\rangle \geq 0\right\}, \\
C_{1, i}=C_{1}=Q_{1}=H, \\
x_{n+1}=P_{C_{n+1} \cap Q_{n+1}}\left(x_{1}\right), \quad \forall n \in \mathbb{N},
\end{array}\right.
$$


where $\theta_{n, i}=\gamma_{n, i} \cdot \Delta_{n}^{2}+\tau_{n, i}(i=1,2, \ldots)$ and $\Delta_{n}=\sup \left\{\left\|x_{n}-z\right\|: z \in \Omega\right\}<\infty$ satisfying the following conditions:

(C1) $\left\{\alpha_{n, i}\right\}_{n=1}^{\infty} \subset\left[a_{1, i}, b_{1, i}\right]$ and $\left\{\beta_{n, i}\right\}_{n=1}^{\infty} \subset\left[a_{2, i}, b_{2, i}\right](i=1,2, \ldots)$ such that $0<a_{j, i}<b_{j, i}<1$ for each $j=1,2$, and $b_{1, i}+b_{2, i}<1-\kappa_{i}(i=1,2, \ldots)$;

(C2) $\lambda \in(0,2 \xi]$ and $\left\{r_{n}\right\}_{n=1}^{\infty} \subset[r, \infty)$ for some $r>0$.

Then the sequence $\left\{x_{n}\right\}_{n=1}^{\infty}$ converges strongly to $w=P_{\Omega}\left(x_{1}\right)$.

If $\Phi=0$, then Corollary 3.3 is reduced immediately to the following result.

Corollary 3.4 Let $H$ be a real Hilbert space, $M: H \rightarrow 2^{H}$ be a maximal monotone mapping, and let $B: H \rightarrow H$ be a $\xi$-cocoercive mapping. For each $i=1,2, \ldots$, let $\left\{T_{i}: H \rightarrow H\right\}$ be an infinite family of uniformly continuous and asymptotically $\kappa_{i}$-SPC mappings in the intermediate sense defined as in (1.18) when $\kappa_{i} \in[0,1)$ with the sequences $\left\{\gamma_{n, i}\right\}_{n=1}^{\infty},\left\{\tau_{n, i}\right\}_{n=1}^{\infty} \subset$ $[0, \infty)$ such that $\lim _{n \rightarrow \infty} \gamma_{n, i}=\lim _{n \rightarrow \infty} \tau_{n, i}=0$. Assume that $\Omega:=\bigcap_{i=1}^{\infty} F\left(T_{i}\right) \cap \operatorname{VI}(H, B, M)$ is a nonempty bounded subset of $H$. For $x_{1}=x \in H$ chosen arbitrarily, suppose that $\left\{x_{n}\right\}_{n=1}^{\infty}$ is generated iteratively by

$$
\left\{\begin{array}{l}
y_{n, i}=\left(1-\alpha_{n, i}-\beta_{n, i}\right) x_{n}+\alpha_{n, i} T_{i}^{n} x_{n}+\beta_{n, i} J_{M, \lambda}\left(x_{n}-\lambda B x_{n}\right), \\
C_{n+1, i}=\left\{z \in C_{n} \cap Q_{n}:\left\|y_{n, i}-z\right\|^{2} \leq\left\|x_{n}-z\right\|^{2}+\theta_{n, i}\right\}, \\
C_{n+1}=\bigcap_{i=1}^{\infty} C_{n+1, i}, \\
Q_{n+1}=\left\{z \in C_{n} \cap Q_{n}:\left\langle x_{n}-z, x_{1}-x_{n}\right\rangle \geq 0\right\}, \\
C_{1, i}=C_{1}=Q_{1}=H, \\
x_{n+1}=P_{C_{n+1} \cap Q_{n+1}}\left(x_{1}\right), \quad \forall n \in \mathbb{N},
\end{array}\right.
$$

where $\theta_{n, i}=\gamma_{n, i} \cdot \Delta_{n}^{2}+\tau_{n, i}(i=1,2, \ldots)$ and $\Delta_{n}=\sup \left\{\left\|x_{n}-z\right\|: z \in \Omega\right\}<\infty$ satisfying the following conditions:

(C1) $\left\{\alpha_{n, i}\right\}_{n=1}^{\infty} \subset\left[a_{1, i}, b_{1, i}\right]$ and $\left\{\beta_{n, i}\right\}_{n=1}^{\infty} \subset\left[a_{2, i}, b_{2, i}\right](i=1,2, \ldots)$ such that $0<a_{j, i}<b_{j, i}<1$ for each $j=1,2$, and $b_{1, i}+b_{2, i}<1-\kappa_{i}(i=1,2, \ldots)$;

(C2) $\lambda \in(0,2 \xi]$.

Then the sequence $\left\{x_{n}\right\}_{n=1}^{\infty}$ converges strongly to $w=P_{\Omega}\left(x_{1}\right)$.

If $B=0$ and $M=0$, then Theorem 3.1 is reduced immediately to the following result.

Corollary 3.5 Let $C$ be a nonempty closed convex subset of a real Hilbert space $H$, $\Phi$ be a bifunction from $C \times C$ into $\mathbb{R}$ satisfying the conditions (A1)-(A5), and let $\varphi: C \rightarrow \mathbb{R} \cup\{+\infty\}$ be a proper lower semicontinuous and convex function with the assumption that either (B1) or (B2) holds. For each $i=1,2, \ldots$, let $\left\{T_{i}: C \rightarrow C\right\}$ be an infinite family of uniformly continuous and asymptotically $\kappa_{i}$-SPC mappings in the intermediate sense defined as in (1.18) when $\kappa_{i} \in[0,1)$ with the sequences $\left\{\gamma_{n, i}\right\}_{n=1}^{\infty},\left\{\tau_{n, i}\right\}_{n=1}^{\infty} \subset[0, \infty)$ such that $\lim _{n \rightarrow \infty} \gamma_{n, i}=$ $\lim _{n \rightarrow \infty} \tau_{n, i}=0$. Assume that $\Omega:=\bigcap_{i=1}^{\infty} F\left(T_{i}\right) \cap \operatorname{MEP}(\Phi, \varphi)$ is a nonempty bounded subset 
of C. For $x_{1}=x \in C$ chosen arbitrarily, suppose that $\left\{x_{n}\right\}_{n=1}^{\infty}$ is generated iteratively by

$$
\left\{\begin{array}{l}
u_{n} \in C \text { such that } \\
\quad \Phi\left(u_{n}, y\right)+\varphi(y)-\varphi\left(u_{n}\right)+\frac{1}{r_{n}}\left\langle y-u_{n}, u_{n}-x_{n}\right\rangle \geq 0, \quad \forall y \in C, \\
y_{n, i}=\left(1-\alpha_{n, i}\right) u_{n}+\alpha_{n, i} T_{i}^{n} u_{n}, \\
C_{n+1, i}=\left\{z \in C_{n} \cap Q_{n}:\left\|y_{n, i}-z\right\|^{2} \leq\left\|x_{n}-z\right\|^{2}+\theta_{n, i}\right\}, \\
C_{n+1}=\bigcap_{i=1}^{\infty} C_{n+1, i}, \\
Q_{n+1}=\left\{z \in C_{n} \cap Q_{n}:\left\langle x_{n}-z, x_{1}-x_{n}\right\rangle \geq 0\right\}, \\
C_{1, i}=C_{1}=Q_{1}=C, \\
x_{n+1}=P_{C_{n+1} \cap Q_{n+1}}\left(x_{1}\right), \quad \forall n \in \mathbb{N},
\end{array}\right.
$$

where $\theta_{n, i}=\gamma_{n, i} \cdot \Delta_{n}^{2}+\tau_{n, i}(i=1,2, \ldots)$ and $\Delta_{n}=\sup \left\{\left\|x_{n}-z\right\|: z \in \Omega\right\}<\infty$ satisfying the following conditions:

(C1) $\left\{\alpha_{n, i}\right\}_{n=1}^{\infty} \subset[a, b](i=1,2, \ldots)$ such that $0<a<b<1-\kappa_{i}$;

(C2) $\left\{r_{n}\right\}_{n=1}^{\infty} \subset[r, \infty)$ for some $r>0$.

Then the sequence $\left\{x_{n}\right\}_{n=1}^{\infty}$ converges strongly to $w=P_{\Omega}\left(x_{1}\right)$.

If $\varphi=0$, then Corollary 3.5 is reduced immediately to the following result.

Corollary 3.6 Let $C$ be a nonempty closed convex subset of a real Hilbert space $H$ and let $\Phi$ be a bifunction from $C \times C$ into $\mathbb{R}$ satisfying the conditions (A1)-(A5) with the assumption that either (B2) or (B3) holds. For each $i=1,2, \ldots$, let $\left\{T_{i}: C \rightarrow C\right\}$ be an infinite family of uniformly continuous and asymptotically $\kappa_{i}$-SPC mappings in the intermediate sense defined as in (1.18) when $\kappa_{i} \in[0,1)$ with the sequences $\left\{\gamma_{n, i}\right\}_{n=1}^{\infty},\left\{\tau_{n, i}\right\}_{n=1}^{\infty} \subset[0, \infty)$ such that $\lim _{n \rightarrow \infty} \gamma_{n, i}=\lim _{n \rightarrow \infty} \tau_{n, i}=0$. Assume that $\Omega:=\bigcap_{i=1}^{\infty} F\left(T_{i}\right) \cap \operatorname{EP}(\Phi)$ is a nonempty bounded subset of $C$. For $x_{1}=x \in C$ chosen arbitrarily, suppose that $\left\{x_{n}\right\}_{n=1}^{\infty}$ is generated iteratively by

$$
\left\{\begin{array}{l}
u_{n} \in C \text { such that } \Phi\left(u_{n}, y\right)+\frac{1}{r_{n}}\left\langle y-u_{n}, u_{n}-x_{n}\right\rangle \geq 0, \quad \forall y \in C, \\
y_{n, i}=\left(1-\alpha_{n, i}\right) u_{n}+\alpha_{n, i} T_{i}^{n} u_{n}, \\
C_{n+1, i}=\left\{z \in C_{n} \cap Q_{n}:\left\|y_{n, i}-z\right\|^{2} \leq\left\|x_{n}-z\right\|^{2}+\theta_{n, i}\right\}, \\
C_{n+1}=\bigcap_{i=1}^{\infty} C_{n+1, i}, \\
Q_{n+1}=\left\{z \in C_{n} \cap Q_{n}:\left\langle x_{n}-z, x_{1}-x_{n}\right\rangle \geq 0\right\}, \\
C_{1, i}=C_{1}=Q_{1}=C, \\
x_{n+1}=P_{C_{n+1} \cap Q_{n+1}}\left(x_{1}\right), \quad \forall n \in \mathbb{N},
\end{array}\right.
$$

where $\theta_{n, i}=\gamma_{n, i} \cdot \Delta_{n}^{2}+\tau_{n, i}(i=1,2, \ldots)$ and $\Delta_{n}=\sup \left\{\left\|x_{n}-z\right\|: z \in \Omega\right\}<\infty$ satisfying the following conditions:

(C1) $\left\{\alpha_{n, i}\right\}_{n=1}^{\infty} \subset[a, b](i=1,2, \ldots)$ such that $0<a<b<1-\kappa_{i}$;

(C2) $\left\{r_{n}\right\}_{n=1}^{\infty} \subset[r, \infty)$ for some $r>0$.

Then the sequence $\left\{x_{n}\right\}_{n=1}^{\infty}$ converges strongly to $w=P_{\Omega}\left(x_{1}\right)$.

If $\Phi=0$, then Corollary 3.6 is reduced immediately to the following result. 
Corollary 3.7 Let C be a nonempty closed convex subset of a real Hilbert space H. For each $i=1,2, \ldots$, let $\left\{T_{i}: C \rightarrow C\right\}$ be an infinite family of uniformly continuous and asymptotically $\kappa_{i}$-SPC mappings in the intermediate sense defined as in (1.18) when $\kappa_{i} \in[0,1)$ with the sequences $\left\{\gamma_{n, i}\right\}_{n=1}^{\infty},\left\{\tau_{n, i}\right\}_{n=1}^{\infty} \subset[0, \infty)$ such that $\lim _{n \rightarrow \infty} \gamma_{n, i}=\lim _{n \rightarrow \infty} \tau_{n, i}=0$. Assume that $\Omega:=\bigcap_{i=1}^{\infty} F\left(T_{i}\right)$ is a nonempty bounded subset of $C$. For $x_{1}=x \in C$ chosen arbitrarily, suppose that $\left\{x_{n}\right\}_{n=1}^{\infty}$ is generated iteratively by

$$
\left\{\begin{array}{l}
y_{n, i}=\left(1-\alpha_{n, i}\right) x_{n}+\alpha_{n, i} T_{i}^{n} x_{n}, \\
C_{n+1, i}=\left\{z \in C_{n} \cap Q_{n}:\left\|y_{n, i}-z\right\|^{2} \leq\left\|x_{n}-z\right\|^{2}+\theta_{n, i}\right\}, \\
C_{n+1}=\bigcap_{i=1}^{\infty} C_{n+1, i}, \\
Q_{n+1}=\left\{z \in C_{n} \cap Q_{n}:\left\langle x_{n}-z, x_{1}-x_{n}\right\rangle \geq 0\right\}, \\
C_{1, i}=C_{1}=Q_{1}=C, \\
x_{n+1}=P_{C_{n+1} \cap Q_{n+1}}\left(x_{1}\right), \quad \forall n \in \mathbb{N},
\end{array}\right.
$$

where $\theta_{n, i}=\gamma_{n, i} \cdot \Delta_{n}^{2}+\tau_{n, i}(i=1,2, \ldots), \Delta_{n}=\sup \left\{\left\|x_{n}-z\right\|: z \in \Omega\right\}<\infty$ and $\left\{\alpha_{n, i}\right\}_{n=1}^{\infty} \subset[a, b]$ $(i=1,2, \ldots)$ such that $0<a<b<1-\kappa_{i}$. Then the sequence $\left\{x_{n}\right\}_{n=1}^{\infty}$ converges strongly to $w=P_{\Omega}\left(x_{1}\right)$.

Recall that for each $i=1,2, \ldots$, a mapping $T_{i}: C \rightarrow C$ is said to be asymptotically nonexpansive if there exists a sequence $\left\{\gamma_{n, i}\right\} \subset[0, \infty)$ with $\lim _{n \rightarrow \infty} \gamma_{n, i}=0$ such that

$$
\left\|T_{i}^{n} x-T_{i}^{n} y\right\| \leq \sqrt{1+\gamma_{n, i}}\|x-y\|, \quad \forall x, y \in C
$$

for all $n \in \mathbb{N}$. If $\kappa_{i}=0$ and $\tau_{n, i}=0$ for all $i=1,2, \ldots$ and $n \in \mathbb{N}$, then Corollary 3.7 is reduced immediately to the following result.

Corollary 3.8 Let C be a nonempty closed convex subset of a real Hilbert space H. For each $i=1,2, \ldots$, let $\left\{T_{i}: C \rightarrow C\right\}$ be an infinite family of asymptotically nonexpansive mappings defined as in (3.37) with the sequence $\left\{\gamma_{n, i}\right\}_{n=1}^{\infty} \subset[0, \infty)$ such that $\lim _{n \rightarrow \infty} \gamma_{n, i}=0$. Assume that $\Omega:=\bigcap_{i=1}^{\infty} F\left(T_{i}\right)$ is a nonempty bounded subset of C. For $x_{1}=x \in C$ chosen arbitrarily, suppose that $\left\{x_{n}\right\}_{n=1}^{\infty}$ is generated iteratively by

$$
\left\{\begin{array}{l}
y_{n, i}=\left(1-\alpha_{n, i}\right) x_{n}+\alpha_{n, i} T_{i}^{n} x_{n}, \\
C_{n+1, i}=\left\{z \in C_{n} \cap Q_{n}:\left\|y_{n, i}-z\right\|^{2} \leq\left\|x_{n}-z\right\|^{2}+\theta_{n, i}\right\}, \\
C_{n+1}=\bigcap_{i=1}^{\infty} C_{n+1, i}, \\
Q_{n+1}=\left\{z \in C_{n} \cap Q_{n}:\left\langle x_{n}-z, x_{1}-x_{n}\right\rangle \geq 0\right\}, \\
C_{1, i}=C_{1}=Q_{1}=C, \\
x_{n+1}=P_{C_{n+1} \cap Q_{n+1}}\left(x_{1}\right), \quad \forall n \in \mathbb{N},
\end{array}\right.
$$

where $\theta_{n, i}=\gamma_{n, i} \cdot \Delta_{n}^{2}(i=1,2, \ldots), \Delta_{n}=\sup \left\{\left\|x_{n}-z\right\|: z \in \Omega\right\}<\infty$, and $\left\{\alpha_{n, i}\right\}_{n=1}^{\infty} \subset[a, b](i=$ $1,2, \ldots)$ such that $0<a<b<1$. Then the sequence $\left\{x_{n}\right\}_{n=1}^{\infty}$ converges strongly to $w=P_{\Omega}\left(x_{1}\right)$. 


\section{Applications}

We introduce the equilibrium problem to the optimization problem:

$$
\min _{x \in C} \zeta(x)
$$

where $C$ is a nonempty closed convex subset of a real Hilbert space $H$ and $\zeta: C \rightarrow \mathbb{R} \cup$ $\{+\infty\}$ is proper convex and lower semicontinuous. We denote by $\operatorname{Argmin}(\zeta)$ the set of solutions of problem (4.1). We define the condition (B4) as the condition (B3) such that $\Phi: C \times C \rightarrow \mathbb{R}$ is a bifunction defined by $\Phi(x, y)=\zeta(y)-\zeta(x)$ for all $x, y \in C$. Observe that $\operatorname{EP}(\Phi)=\operatorname{Argmin}(\zeta)$. We obtain that Corollary 3.3 is reduced immediately to the following result.

Theorem 4.1 Let $H$ be a real Hilbert space and let $\zeta: H \rightarrow \mathbb{R} \cup\{+\infty\}$ be a proper lower semicontinuous and convex function with the assumption that either (B2) or (B4) holds. Let $M: H \rightarrow 2^{H}$ be a maximal monotone mapping and let $B: H \rightarrow H$ be a $\xi$-cocoercive mapping. For each $i=1,2, \ldots$, let $\left\{T_{i}: H \rightarrow H\right\}$ be an infinite family of uniformly continuous and asymptotically $\kappa_{i}$-SPC mappings in the intermediate sense defined as in (1.18) when $\kappa_{i} \in$ $[0,1)$ with the sequences $\left\{\gamma_{n, i}\right\}_{n=1}^{\infty},\left\{\tau_{n, i}\right\}_{n=1}^{\infty} \subset[0, \infty)$ such that $\lim _{n \rightarrow \infty} \gamma_{n, i}=\lim _{n \rightarrow \infty} \tau_{n, i}=0$. Assume that $\Omega:=\bigcap_{i=1}^{\infty} F\left(T_{i}\right) \cap V I(H, B, M) \cap \operatorname{Argmin}(\zeta)$ is a nonempty bounded subset of $H$. For $x_{1}=x \in H$ chosen arbitrarily, suppose that $\left\{x_{n}\right\}_{n=1}^{\infty}$ is generated iteratively by

$$
\left\{\begin{array}{l}
u_{n} \in H \text { such that } \zeta(y)-\zeta\left(u_{n}\right)+\frac{1}{r_{n}}\left\langle y-u_{n}, u_{n}-x_{n}\right\rangle \geq 0, \quad \forall y \in H, \\
y_{n, i}=\left(1-\alpha_{n, i}-\beta_{n, i}\right) u_{n}+\alpha_{n, i} T_{i}^{n} u_{n}+\beta_{n, i} J_{M, \lambda}\left(u_{n}-\lambda B u_{n}\right), \\
C_{n+1, i}=\left\{z \in C_{n} \cap Q_{n}:\left\|y_{n, i}-z\right\|^{2} \leq\left\|x_{n}-z\right\|^{2}+\theta_{n, i}\right\}, \\
C_{n+1}=\bigcap_{i=1}^{\infty} C_{n+1, i}, \\
Q_{n+1}=\left\{z \in C_{n} \cap Q_{n}:\left\langle x_{n}-z, x_{1}-x_{n}\right\rangle \geq 0\right\}, \\
C_{1, i}=C_{1}=Q_{1}=H, \\
x_{n+1}=P_{C_{n+1} \cap Q_{n+1}}\left(x_{1}\right), \quad \forall n \in \mathbb{N},
\end{array}\right.
$$

where $\theta_{n, i}=\gamma_{n, i} \cdot \Delta_{n}^{2}+\tau_{n, i}(i=1,2, \ldots)$ and $\Delta_{n}=\sup \left\{\left\|x_{n}-z\right\|: z \in \Omega\right\}<\infty$ satisfying the following conditions:

(C1) $\left\{\alpha_{n, i}\right\}_{n=1}^{\infty} \subset\left[a_{1, i}, b_{1, i}\right]$ and $\left\{\beta_{n, i}\right\}_{n=1}^{\infty} \subset\left[a_{2, i}, b_{2, i}\right](i=1,2, \ldots)$ such that $0<a_{j, i}<b_{j, i}<1$ for each $j=1,2$, and $b_{1, i}+b_{2, i}<1-\kappa_{i}(i=1,2, \ldots)$;

(C2) $\lambda \in(0,2 \xi]$ and $\left\{r_{n}\right\}_{n=1}^{\infty} \subset[r, \infty)$ for some $r>0$.

Then the sequence $\left\{x_{n}\right\}_{n=1}^{\infty}$ converges strongly to $w=P_{\Omega}\left(x_{1}\right)$.

Competing interests

The author declares that he has no competing interests.

\section{Acknowledgements}

The author would like to thank the Faculty of Science, Maejo University for its financial support.

Received: 2 May 2012 Accepted: 7 November 2012 Published: 26 November 2012

\section{References}

1. Takahashi, W: Nonlinear Functional Analysis. Yokohama Publishers, Yokohama (2000)

2. Browder, FE, Petryshyn, WV: Construction of fixed points of nonlinear mappings in Hilbert spaces. J. Math. Anal. Appl. 20, 197-228 (1967) 
3. Qihou, L: Convergence theorems of the sequence of iterates for asymptotically demicontractive and hemicontractive mappings. Nonlinear Anal. 26, 1835-1842 (1996)

4. Goebel, K, Kirk, WA: A fixed point theorem for asymptotically nonexpansive mappings. In: Proceedings of the American Mathematical Society, vol. 35, pp. 171-174 (1972)

5. Osilike, MO, Udomene, A, Igbokwe, DI, Akuchu, BG: Demiclosedness principle and convergence theorems for k-strictly asymptotically pseudocontractive maps. J. Math. Anal. Appl. 326, 1334-1345 (2007)

6. Sahu, DR, Xu, HK, Yao, JC: Asymptotically strict pseudocontractive mappings in the intermediate sense. Nonlinear Anal. 70, 3502-3511 (2009)

7. $\mathrm{Hu}, \mathrm{CS}, \mathrm{Cai}, \mathrm{G}$ : Convergence theorems for equilibrium problems and fixed point problems of a finite family of asymptotically k-strictly pseudocontractive mappings in the intermediate sense. Comput. Math. Appl. 61, 79-93 (2011)

8. Duan, P, Zhao, J: Strong convergence theorems for system of equilibrium problems and asymptotically strict pseudocontractions in the intermediate sense. Fixed Point Theory Appl. 2011, 13 (2011)

9. Ezeora, JN, Shehu, Y: Strong convergence theorems for asymptotically strict pseudocontractive mappings in the intermediate sense. Thai J. Math. 9(2), 399-409 (2011)

10. Ge, CS: Strong convergence of iterative algorithms with variable coefficients for asymptotically strict pseudocontractive mappings in the intermediate sense and monotone mappings. Fixed Point Theory Appl. 2012,68 (2012)

11. Ceng, LC, Yao, JC: Strong convergence theorems for variational inequalities and fixed point problems of asymptotically strict pseudocontractive mappings in the intermediate sense. Acta Appl. Math. (2011). doi:10.1007/s10440-011-9614-x

12. Zhao, J, He, S, Dong, QL: Strong convergence theorems for generalized equilibrium problems, variational inequality and fixed point problems of asymptotically strict pseudocontractive mappings in the intermediate sense. Thai J. Math. 9(2), 267-283 (2011)

13. Qin, X, Kim, JK, Wang, T: On the convergence of implicit iterative processes for asymptotically pseudocontractive mappings in the intermediate sense. Abstr. Appl. Anal. (2011). doi:10.1155/2011/468716

14. Mann, WR: Mean value methods in iteration. In: Proceedings of the American Mathematical Society, vol. 4, pp. 506-510 (1953)

15. Kim, TH, Xu, HK: Strong convergence of modified Mann iterations. Nonlinear Anal. 61, 51-60 (2005)

16. Reich, S: Weak convergence theorems for nonexpansive mappings in Banach spaces. J. Math. Anal. Appl. 67, 274-276 (1979)

17. Marino, G, Xu, HK: Convergence of generalized proximal point algorithms. Commun. Pure Appl. Anal. 3, 791-808 (2004)

18. Kim, TH, Xu, HK: Convergence of the modified Mann's iteration method for asymptotically strict pseudo-contractions Nonlinear Anal. 68, 2828-2836 (2008)

19. Genel, A, Lindenstrauss, J: An example concerning fixed points. Isr. J. Math. 22, 81-86 (1975)

20. Nakajo, K, Takahashi, W: Strong convergence theorems for nonexpansive mappings and nonexpansive semigroups. J. Math. Anal. Appl. 279, 372-379 (2003)

21. Marino, G, Xu, HK: Weak and strong convergence theorems for strict pseudo-contractions in Hilbert spaces. J. Math. Anal. Appl. 329, 336-346 (2007)

22. Blum, E, Oettli, W: From optimization and variational inequalities to equilibrium problems. Math. Stud. 63, 123-145 (1994)

23. Colao, V, Marino, G, Xu, HK: An iterative method for finding common solutions of equilibrium and fixed point problems. J. Math. Anal. Appl. 344, 340-352 (2008)

24. Tianchai, P: Shrinking projection method of common fixed point problems for discrete asymptotically strictly pseudocontractive semigroups and mixed equilibrium problems in Hilbert spaces. ISRN Math. Anal. 2011, Article ID 529407 (2011)

25. Peng, JW, Yao, JC: Two extragradient methods for generalized mixed equilibrium problems, nonexpansive mappings and monotone mappings. J. Comput. Appl. Math. 58, 1287-1301 (2009)

26. Duan, PC: Convergence theorems concerning hybrid methods for strict pseudocontractions and systems of equilibrium problems. J. Inequal. Appl. 2010, 396080 (2010)

27. Flam, SD, Antipin, AS: Equilibrium programming using proximal-like algorithms. Math. Program. 78, 29-41 (1997)

28. Cho, YJ, Kang, Jl, Qin, X: Convergence theorems based on hybrid methods for generalized equilibrium problems and fixed point problems. Nonlinear Anal. 71, 4203-4214 (2009)

29. Cho, YJ, Argyros, IK, Petrot, N: Approximation methods for common solutions of generalized equilibrium, systems of nonlinear variational inequalities and fixed point problems. Comput. Math. Appl. 60, 2292-2301 (2010)

30. Cho, YJ, Petrot, N: On the system of nonlinear mixed implicit equilibrium problems in Hilbert spaces. J. Inequal. Appl. 2010, Article ID 437976 (2010). doi:10.1155/2010/437976

31. Cho, YJ, Petrot, N: An optimization problem related to generalized equilibrium and fixed point problems with applications. Fixed Point Theory Appl. 11, 237-250 (2010)

32. He, H, Liu, S, Cho, YJ: An explicit method for systems of equilibrium problems and fixed points of infinite family of nonexpansive mappings. J. Comput. Appl. Math. 235, 4128-4139 (2011)

33. Qin, X, Chang, SS, Cho, YJ: Iterative methods for generalized equilibrium problems and fixed point problems with applications. Nonlinear Anal., Real World Appl. 11, 2963-2972 (2010)

34. Ceng, LC, Guu, SM, Yao, JC: Hybrid iterative method for finding common solutions of generalized mixed equilibrium and fixed point problems. Fixed Point Theory Appl. 2012, 92 (2012). doi:10.1186/1687-1812-2012-92

35. Balocchi, C, Capelo, A: Variational and Quasivariational Inequalities, Applications to Free Boundary Problems. Wiley, New York (1984)

36. Isac, G, Bulavsky, VA, Kalashnikkov, VV: Complementarity, Equilibrium, Efficiency and Economics. Kluwer Academic, Dordrecht (2002)

37. Konnov, I: Combined Relaxation Methods for Variational Inequalities. Lecture Notes in Economics and Math. Systems, vol. 495. Springer, Berlin (2001) 
38. Kinderlerer, D, Stampaccia, G: An Introduction to Variational Inequalities and Their Applications. Academic Press, New York (1980)

39. Nagurney, A: Network Economics a Variational Inequality Approach. Kluwer Academic, Dordrecht (1993)

40. Panagiotoupoulos, PD, Stavroulakis, GE: New types of variational principles based on the notion of quasidifferentiability. Acta Mech. 94, 171-194 (1992)

41. Patriksson, M: Nonlinear Programming and Variational Inequality Problems. A Unified Approach. Kluwer Academic, Dordrecht (1999)

42. Zhang, SS, Lee, JHW, Chan, CK: Algorithms of common solutions to quasi variational inclusion and fixed point problems. Appl. Math. Mech. 29(5), 571-581 (2008)

43. Qin, X, Cho, YJ, Kang, SM, Shang, M: A hybrid iterative scheme for asymptotically $k$-strict pseudo-contractions in Hilbert spaces. Nonlinear Anal. 70, 1902-1911 (2009)

44. Kim, TH: Convergence of modified Mann's iteration methods for discrete asymptotically strict pseudo-contractive semigroups. In: Proceedings of the Asian Conference on Nonlinear Analysis and Optimization, pp. 139-162 (2008)

45. Ishikawa, S: Fixed points by a new iteration method. Proc. Am. Math. Soc. 44(1), 147-150 (1974)

46. Osilike, $\mathrm{MO}$, Igbokwe, Dl: Weak and strong convergence theorems for fixed points of pseudocontractions and solutions of monotone type operator equations. J. Comput. Appl. Math. 40, 559-567 (2000)

47. Brézis, H: Opérateurs Maximaux Monotones et Semi-Groupes de Contractions dans les Espaces de Hilbert. North-Holland Math Stud., vol. 5. North-Holland, Amsterdam (1973). (Notas de Matemática (50))

doi:10.1186/1687-1812-2012-214

Cite this article as: Tianchai: An approximate solution to the fixed point problems for an infinite family of asymptotically strictly pseudocontractive mappings in the intermediate sense, cocoercive quasivariational inclusions problems and mixed equilibrium problems in Hilbert spaces. Fixed Point Theory and Applications 2012 2012:214.

\section{Submit your manuscript to a SpringerOpen ${ }^{\circ}$ journal and benefit from:}

- Convenient online submission

- Rigorous peer review

- Immediate publication on acceptance

- Open access: articles freely available online

- High visibility within the field

- Retaining the copyright to your article 